\title{
Holocene transgression recorded by sand composition in the mesotidal Galician coastline (NW Spain)
}

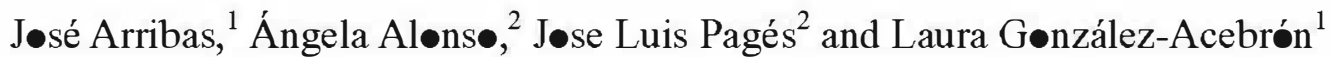

\begin{abstract}

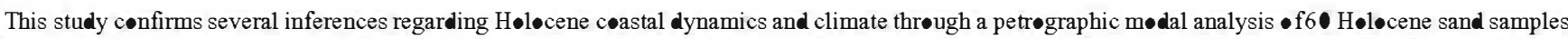

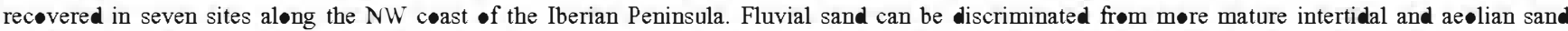

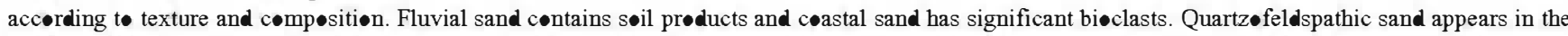

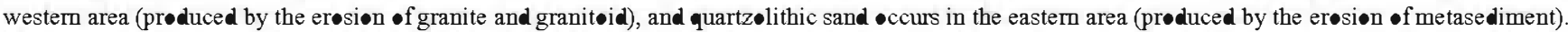

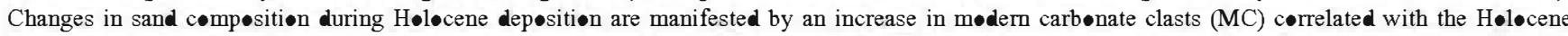

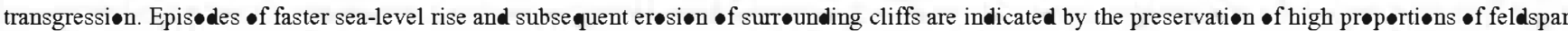
in intertidal sand. In contrast, fluvial sand is characterized by greater quartz enrichment. These inferences were confirmed by petrographic indices (carbønate

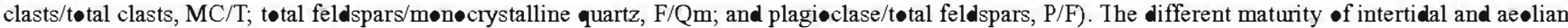

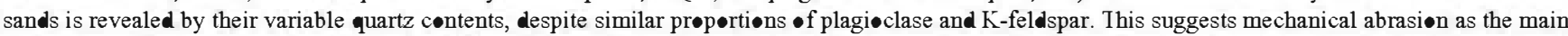

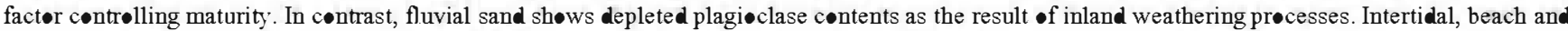

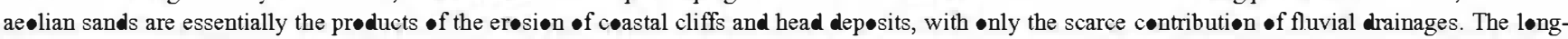

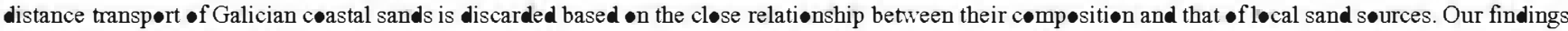

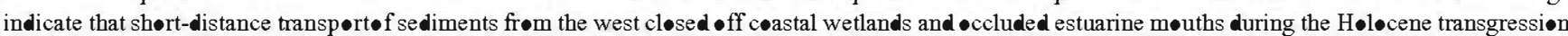

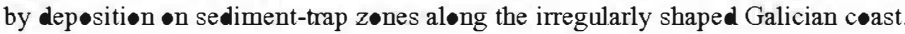

\section{Keywords}

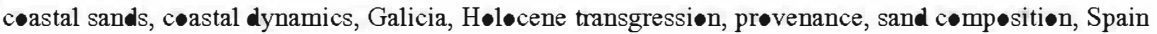

\section{Introduction}

The genesis of recent and modern (i.e. Holocene) deposits has recently generated worldwide interest, since understanding related processes will allow us to more reliably predict future changes. During the last few decades, special efforts have been made to identify climate fluctuations and their consequences in fields related to vegetation (Birks, 2003; Froyd, 2005), micropaleontology (e.g. Anderson, 1995; Smol and Cumming, 2000), isotopes (e.g. Hong et al., 1995) and sedimentology (e.g. Culver et al., 2007). In these fields, sensitive signatures are used as climate indicators.

The northem coast of Galicia and westem area of Asturias, in the northwest sector of the Iberian Peninsula (Figure 1), is an abrupt mesotidal wave-dominated coast, where sedimentation shows a discontinuous distribution in estuaries, barriers, coastal lagoons, beaches and pocket beaches. The Holocene record has been mainly interpreted as infilling sequences of estuaries or back-barrier coastal wetlands (Alonso and Pagés, 2000; García Antón et al., 2006; Bao et al., 2007).

The composition of clastic deposits is the final outcome of the cumulative effects of highly interrelated factors (Johnsson, 1993) that mainly involve source lithology, chemical weathering, hydraulic sorting and abrasion. Thus, detrital modes can be used to decipher these factors. Studies on modem sand composition enable direct comparison between the source system and the sandy product (Ibbeken and Schleyer, 1991; Arribas and Tortosa, 2003) and also serve to monitor other factors. Climate and vegetation control the intensity of chemical weathering of the source rock (e.g. Nestbitt et al., 1997). Physiography substantially affects denudation rates, and therefore, the weathering interval (e.g Grantham and Velbel, 1988; Johnsson et al., 1991). Some minerals and clastic particles such as feldspars or rock fragments are sensitive to climate conditions (e.g. Krynine, 1950; Todd, 1968; Suttner and Dutta, 1986), and thus, the bulk composition of sands could be used as a climate proxy.

To address the role of factors such as source area lithology, sedimentary environment and climate on sand composition, this paper describes sand composition and textures of Holocene deposits recorded at seven sites of the northwestern coast of the Iberian Peninsula (Traba, Baldaio, Pto. Coruña, Miño, Pantín, Viveiro and

\footnotetext{
${ }^{1}$ Universidad C•mplutense de Madrid - IGE, C.S.I.C., Spain

${ }^{2}$ Universidad de A Coruña, Spain
}

\section{Corresponding author:}

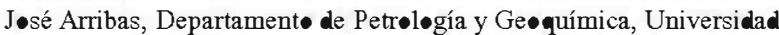
C•mplutense de Madrid - IGE, C.S.I.C., 28040-Madrid, Spain Email: arribas@ge•.ucm.es 


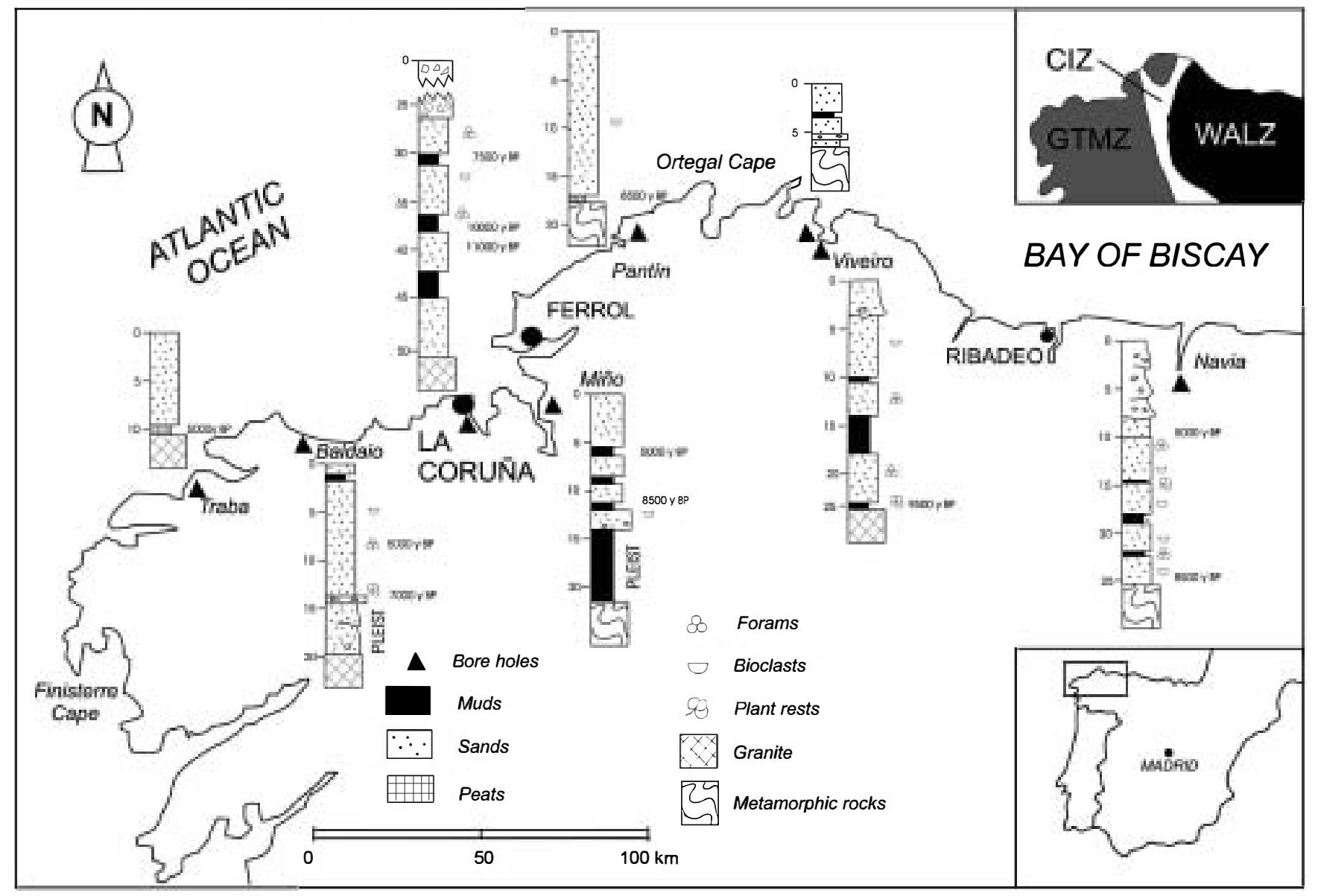

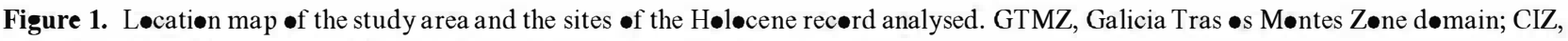

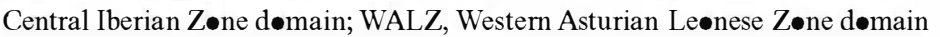

Navia, Figure 1). Further insight into Holocene processes was obtained by comparing our results with those of other studies of the same stratigraphic records (e.g. Alonso and Pagés, 2000; Alonso et al., 2003; Delgado et al., 2003; Pagés et al., 2003; García Antón et al., 2006; Bao et al., 2007).

\section{General setting}

\section{Climate, waves, tides and sea level}

The climate of the coastal area of Galicia and Asturias is oceanic with abundant regular rainfall (mean 1000 to $2000 \mathrm{~mm} / \mathrm{yr}$ ), and mild temperatures that vary within a narrow range (from averages of $8-9^{\circ} \mathrm{C}$ in January to $18-; 19^{\circ} \mathrm{C}$ in July). Wet warm winds from the west are dominant (Castillo Rodríguez et al., 2006). According to these climate conditions, mechanical and chemical weathering can be considered as moderate (Wilson, 1969).

From winter to summer, wave energy varies greatly. The average annual significant height ( $\mathrm{Hs}$ ) is $2.7 \mathrm{~m}$, and the peak period (Tp) is $11.1 \mathrm{~s}$. Wave provenance remains rather constant throughout the year, with $75 \%$ arising in the W-NNW sector (data from the directional Sea Watch Buoy of Estaca de Bares, located at $44^{\bullet} 3.9^{\prime} \mathrm{N}, 7^{\bullet} 37.1^{\prime} \mathrm{W}$, with a sea floor depth of $382 \mathrm{~m}$; http://www.puertos.es/es/oceanografia_y_meteorologia/banco de_datos/oleaje.html).
The Galician coast is a mesotidal wave-dominated coast with maximum tides of $4.40 \mathrm{~m}$ recorded in springtime (data from Gijón seaport, year 2002). Average rates of sea-level change can be obtained from the records of tide gauges installed along the northern coast of Spain (available through the web page of the Permanent Service of Mean Sea Level, http://www.pol.ac.uk/psmsl/datainfo). In the Atlantic, these sea-level rises have been estimated at 1.5 $\mathrm{mm} / \mathrm{yr}$ in A Coruña during the last 62 available years (1944 2005), to $2.6 \mathrm{~mm} / \mathrm{yr}$ in Vigo during the period 1944-2001 (58 years). In the Bay of Biscay (Cantabrian), Santander seaport records a sea-level rise of $2.2 \mathrm{~mm} / \mathrm{yr}$ for the period 1944-2001 (57 years). These data are slightly higher than averages for northern Europe (1 to $2.5 \mathrm{~mm} / \mathrm{yr}$; Zerbini, 2000).

\section{Physiography and lithology}

The northwest coast of Spain (Galicia) shows a rugged morphology, with high promontories, cliffs up to $100 \mathrm{~m}$ high, headlands, embayed beaches and funnel-shaped incisions (rias). The coast is developed on igneous and metamorphic rocks of the Variscan Chain (Macizo Ibérico) and shows several domains derived from a highly complex collision orogen (e.g. Arenas et al., 1986). Three main domains are represented: (1) the Galicia Tras os Montes Zone (GTMZ), (2) the Central Iberian Zone (CIZ), and (3) the Western Asturleonese Zone (WALZ) (Figure 1). 
Table 1. Sizes of the drainage basins and surface area percentages of their representative lithologies

\begin{tabular}{|c|c|c|c|c|c|c|}
\hline & Total $\left(\mathrm{km}^{2}\right)$ & 1 & 2 & 3 & 4 & 5 \\
\hline Traba & 18.82 & 100.0 & 0.0 & 0.0 & 0.0 & 0.0 \\
\hline Baldai• & 41.13 & 74.0 & 10.9 & 8.1 & 7.1 & $\bullet .0$ \\
\hline Pt•. & 266.15 & 19.7 & 0.0 & 80.3 & 0.0 & $\bullet .0$ \\
\hline Miñ• & 47.90 & 54.3 & 7.1 & 35.5 & 3.1 & $\bullet .0$ \\
\hline Pantín & 8.55 & 12.4 & 50.2 & 37.5 & $\bullet .0$ & 0.0 \\
\hline Viver• & 298.50 & 47.1 & 0.0 & 45.8 & 7.2 & $\bullet .0$ \\
\hline Navia & 1906.56 & $\bullet .6$ & 0.0 & 48.4 & 49.4 & 1.6 \\
\hline
\end{tabular}

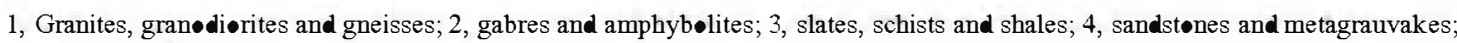

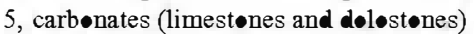

The eastern zone of the area of study corresponds to the westem limit of the Gulf of Biscay. This coastal segment is an E-W straight coast composed of low-grade Cambro-Ordovician metamorphicrocks (schists, quartzites, shales and sandstones) from the WALZ domain. One of the most significant fluvial courses is the Navia River, which is $105 \mathrm{~km}$ long. The drainage basins of these rivers are develope on the Cambro- rdovician WALZ domain.

The Galician coast is an abruptly shaped coastline with dominant NW-SE and NE-SW orientations and high cliffs. Its substrate is composed of Cambro-Ordovician and Silurian metasediments and granites from the WALZ domain, gneisses and quartzites from the $\mathrm{CIZ}$ zone, and granites, gneisses, mafic and ultramafic rocks from the GTMZ domain, in the westernmost area of study. Rivers are no more than $50 \mathrm{~km}$ long, and frequently form rias at their mouths. Inland drainage areas are mainly made up of granites and locally of metasediments and mafic metamorphic rocks.

Drainage basins from the inland fluvial courses of the different sampling sites are highly variable in surface area and percentage drained lithologies (Table 1). Those of Navia, Vivero, Miño and Pto. Coruña are wider drainage basins. Each has a main collector showing constant flow. On the contrary, the smaller catchments (Traba, Baldaio and Pantín) are drained by short streams showing sporadic flow. Coarse-grained rocks ( 1 and 2 in Table 1), especially granites, are the most abundant potential sources of detritus to coastal sand at the respective sampling sites.

\section{Sedimentary record}

The morphology of the coastline is predominantly inherited from former high stands. During the last glacial period, the incision of fluvial systems was developed, leading during the Holocene flooding to the formation of rías, and displaying a wide outer marine zone and an inner estuary. The coastal plain was flooded during the postglacial period, and estuaries were already inundated the beginning of the Holocene. The sea-level rise was fast during the early Holocene (up to $5 \mathrm{~mm} / \mathrm{yr}$ from 8500 to 7500 cal. yr BP), decelerating thereafter $(3.5$ to $2 \mathrm{~mm} /$ $\mathrm{yr}$ in the period 7500 to $5000 \mathrm{yr}$ cal. BP) until its almost stabilization (Alonso et al., 2003; Pagés et al., 2003, 2005). This deceleration induced the replenishment of estuaries and the formation of several beach-barrier complexes in the most open areas of the coast and on the outer parts of the rías. This process caused the concurrent erosion of former sedimentary units in open coasts and cliffs (Lorenzo et al., 2007). Sea-level rise continued thereafter (up to $1.5 \mathrm{~mm} / \mathrm{yr}$ ).
As a consequence, Quatemary outcrops are scarce and appear only as disconnected remains (palaeobeaches and head deposits of Late Pleistocene age, aeolian sands, peats and flash-flood deposits of the Holocene). wing to the scarcity of Holocene outcrops, this work is based on cores obtained in estuaries (Navia, Vivero), barrierlagoon complexes (Miño, Pantín, Baldaio, Traba) and the outer marine zone of the ría of Ares (Pto. Coruña). Boreholes drilled the entire sedimentary cover, as far as the granites and metamorphic rocks comprising the basement (Figure 1). Radiocarbon dating demonstrated that the Late Pleistocene is represented in the bases of three of the cores. These cores, designated according to their corro sponding locations, were Baldaio, in which fluviatile conglomerates and sands in upwards fining sequences appeared; Miño, showing muddy facies and intercalated immature conglomerates representing flash-flood channels in flood flats; and Pto. Coruña, containing fluviatile immature conglomerates and muds, which in the upper portion were dated as Younger Dryas.

In some of the boreholes, the entire Holocene sequence is represented. The Holocene record in Navia and Vivero indicates the replenishment of inner estuaries that were flooded at the beginning of the Holocene. Facies correspond to intertidal mixed flats passing upward in Navia to a fluvial-dominated estuary. Baldaio and Miño correspond to tidal flats in a back-barrier, very shallowlagoon environment, while Pantín and Traba can be considered as coastal lakes or back-dune wetlands with no or only a minor connection to the sea throughout their history. At Pto. Coruña, only the early Holocene is represented; sedimentation took place in tidal flats and intertidal channels developed on the wide platform formed during low sea-level stages and were inundated during the first stages of postglacial flooding.

\section{Petrographic procedures and grain types}

Sixty sand samples were collected from eight mechanical cores drilled on the coastal wetlands of Traba $(10.6 \mathrm{~m})$ and Pantín $(16.5 \mathrm{~m})$; the shallow lagoon complexes of Baldaio $(19.6 \mathrm{~m})$ and Miño $(21.9 \mathrm{~m})$, the estuaries of Vivero $(26.1 \mathrm{~m})$ and Navia $(25.2 \mathrm{~m})$, and in the outer part of the Ria de Ares (Pto. Coruña, $51.5 \mathrm{~m}$ ) (Figure 1). These cores were radiocarbon-dated at several depths, providing ages from $43000 \mathrm{BP}$ (Miño) and $5000 \mathrm{BP}$ (Traba) to the present.

Sands were washed with fresh water to eliminate salts, and wet sieved across the range $2-0.062 \mathrm{~mm}$ to remove mud particles $(<0.062 \mathrm{~mm})$ and scarce granules $(>2 \mathrm{~mm})$. Samples with 
high quantities of organic matter were etched with diluted $\mathrm{H}_{2}$ The sand fraction was air-dried, artificially cemented with epoxy resin, and thin-sectioned. Each thin section was etched with HF and stained by immersion in Na-cobaltinitrite solution to identify feldspars (Chayes, 1952). Alizarine red-S and ferricyanide solution (Lindholm and Finkelman, 1972) were used for carbonate identification. Textures of the sediments (grain size, sorting and quartz grain roundness) were estimated on thin sections using comparative schemes. A petrographic data base was established by counting more than 300 grains in each thin section according to the Gazzi-Dickinson (G-D) method (Ingersoll et al., 1984). Data were tabulated following Zuffa (1985), which allows the ambivalent treatment of data as both the traditional and G-D method. Twenty-eight grain classes were considered and grouped into three main categories: (1) AN, ancient noncarbonates; (2) MC, modern carbonates; and (3) MN, modem non-carbonates.

Ancient non-carbonates (AN) are the most abundant grain type in the sands; this category includes quartz grains (mono- and polycrystalline types), monocrystalline K-feldspars and albites. Twinned grains of plagioclases are commonly both as fresh and variably altere to clay minerals. Micas (muscovite, biotite and chlorite) are very abundant locally. All AN grains occur as monominerals and as the crystal constituents of phaneritic rock fragments (i.e. granites, gneisses) (Table 2). Aphanitic rock fragments ( $\mathrm{L}$ grains in Table 2) in some samples are abundant (e.g. in Navia) and consist of shale, phyllite and chert. In addition, dense minerals may occur as associations comprising tourmaline, zircon, apatite, gamet, homblende, brookite, rutile, anatase and opaque minerals that characterize felsic plutonic sources (Pettijohn et al., 1972). In some samples, these minerals are very abundant and account for $5 \%$ of the total.

Modem carbonates (MC) are represented by bioclasts, mainly of molluscs (abraded lamellibranchian shells and a few unspecified gastropods); echinoderms, foraminifera, red algae, bryozoans and ostracods also appear but in lower proportions. Commonly, shells are micritized, which makes their identification difficult. Locally, this category is highly represented in the sands and amounts to $58 \%$ of total grains. In some samples, these grains are absent.

Modem non-carbonates (MN) consist mainly of silty-clayey grains with irregular edges and large grain sizes. Concretions of Fe-oxides (alterites) are locally abundant (5\%), representing clear land side supplies. These grains occur in sands related to the infill of channels from intertidal mixed flats. Glauconite was identified as trace grains $(<1 \%)$ in sands from the Miño record.

Several compositional parameters and indices have been considered to describe the modal composition of sands using binary (carbonate to total grains $-\mathrm{C} / \mathrm{T}-$ versus ancient non-carbonates to total grains - AN/T -, modified from Di Giulio and Valloni, 1992) and ternary diagrams (QFR, Pettijohn et al., 1972; QmFLt and QmKP, Dickinson et al., 1983; and Qmremoep, according to the criteria of Basu et al., 1975). In addition, indices such as $\mathrm{Rg} / \mathrm{Rt}$ (coarse crystalline rock fragments/total rock fragments), F/Qm (feldspars/monocrystalline quartz), and P/F (plagioclase/ feldspars) were calculated to clarify variations in sand composition through time.

Point-count raw data and recalculated variables are shown in Table 2

\section{Textures of sands}

Grain sizes in the sand deposits vary according to the environment (Table 3). Aeolian and intertidal deposits show homogeneous grain-size intervals, mainly from 177 to $350 \mu \mathrm{m}$. These consist of unimodal deposits, which are well or very well sorted with large populations of high quartz roundness. Bimodal sands are also present and were interpreted as the result of mixing of aeolian and intertidal sands. Grain size decreases where intertidal environments are related to mixed flats, or may increase in association with fluvial channel infill. In both cases, sorting decreases and the angular quartz grain population stabilizes (Table 3 ).

Fluvial deposits show wider grain size intervals; from very fine (fluvial flats or swamps areas) to very coarse sands (lag deposits in channels). These deposits are very poorly sorted and quartz roundness is low. The lack of roundness in quartz grains is, therefore, indicative of sand directly arising from the drainage basin.

Locally, embayed quartz grains were identified in fluvial channel sands in association with alterite grains, suggesting an origin related to weathering profiles formed in drainage areas (Cleary and Conolly, 1971; Johnsson, 1990).

\section{Composition of sands}

The composition of the total grain population (Figure 2) is siliciclastic (AN) with variable amounts of bioclastic carbonate grains (MC) that usually do not excee $25 \%$ of total clasts. The ancient siliciclastic character of the sand reflects an origin related to main land supplies from the erosion of drainage areas and sea cliffs, with the scarce contribution of modern carbonate bioclasts. Hybrid sand containing more than $25 \%$ bioclasts is also observed in the Pantín, Baldaio and Navia records. Mixing between these two end-member contributors of sand is reflected in Figure 2 in the continuous plots of sands along the diagonal line of the diagram. Sand from Pto. Coruña differs from this general distribution pattern in that it shows the presence of substantial amounts of modem silty-clayey drapes (MN).

The absence of bioclasts in sand can be correlated with fluvial deposits, as occurs at the base of the successions of Pto. Coruna, Miño, Baldaio and Vivero-Puente. At the top of this last section, bioclasts are also absent. In aeolian deposits (mainly in Traba, and Pantín), the content of bioclasts is low and almost constant through the sections. Intertidal deposits (e.g. Navia, top of Pto. Coruña, and top of Baldaio sections) show more scattered bioclast contents, although these increase towards the top of the sequences (Pto. Coruña and Baldaio)

\section{Main modal sand composition (QFR; QmFLt)}

The ancient siliciclastic components (AN) of all the analysed sands of the Galician coast are quartzofeldspathic. This character is manifeste in the sand plots close to the QF line in the QFR diagram; and even closer to the line in the emFLt diagram (Figure 3). Differences between these two diagrams reflect the presence of coarse-grained rock fragments in the sands. Quartzofeldspathic petrofacies reflect an origin related to erosion of coarse crystalline rocks (granitoid) that constitute the main lithology of the coastal cliffs and drainage basins (CIZ of the Iberian Massif). 
Traba

\begin{tabular}{lllll}
\hline TRA-9,60 TRA-8,8 TRA-6,60 & TRA-5,2 & TRA-3,2 & TRA- $\mathbf{0 , 8 \bullet}$ \\
\hline
\end{tabular}

AN

Q

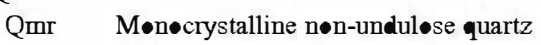

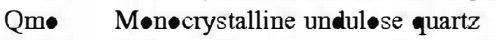

Qp2-3 P•lycrystalline quartz (2-3 crystals)

$\mathrm{Qp}>3 \quad$ P $\bullet$ lycrystalline quartz (>3 crystals)

Qrm Quartz in low and medium meta. grade reck fragm.

Qrp Quartz in plut॰nic røck fragment

Qrg Quartz in gneissic røck fragment

Qrs Quartz in sandstone

$\mathrm{K}$

Ks $\quad \mathrm{K}$-feldspar (single crystal)

Krm K-feldspar in løw and medium meta. grade røck fragm.

Krp K-feldspar in plutønic reck fragment

Krg K-feldspar in gneissic røck fragment

$\mathrm{P}$

Ps Plagiøclase (single crystal)

Prm Plagiøclase in løw and medium meta. grade rock fragm.

Prp Plagiøclase in plut•nic røck fragment

$\mathrm{M}$

$\mathrm{Bi} \quad$ Bi॰tite (single crystal)

$\mathrm{Mu} \quad$ Musc $\bullet$ vite (single crystal)

147

39

21

4

1

31

2

19

Chlørite (single crystal)

Mrm Mica in løw and medium meta. grade reck fragm.

L

$\mathrm{Ch} \quad$ Unspecified chert

Sh Shale and fillites

Dm Dense minerals (unspecified)

MC

MN

Mølusc

Echin॰derm

F•ram.; red algae; bri $\bullet \bullet \bullet$;

-straceds

Indeterminate biøclast

13

4

22

Silty-clay søft grains,

glauc»nite

Fe-Oxides, alterite

Total

$\mathrm{MC} / \mathrm{T}$

AN/T

Q

F

$\mathrm{R}$

Qm

F

Lt

Qm

$\mathrm{K}$
$\mathrm{P}$

Qmr

Qm•

Qp

$\mathrm{Rg} / \mathrm{Rt}$

$\mathrm{F} / \mathrm{Qm}$

$\mathrm{P} / \mathrm{F}$

2

1.1

80.3

19.7

0.0

12.5

7.2

11.8

$\begin{array}{rr}130 & 128 \\ 54 & 52 \\ 15 & 8 \\ 9 & 13\end{array}$

143

40

17

13

3

3

43

31

37

128

50

12

11

131

61

7

11

4

(39.

25

27

18

21

27

28

1

1

1

1

$\begin{array}{rr}16 & 23 \\ 1 & 3\end{array}$

26
3

18

10

14

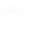

12

10

309

310

303

306

0.13

0.87

0.09

0.12

0.91

0.88

0.13

.12

0.88

0.09

$79.9 \quad 74.6$

74.2

77.1

75.0

0.91

23.7

22.5

1.8

19.2

23.9

75.5

3.3

75.3

75.6

24.4

24.7

0.0

3.7

1.1

22.3

2.2

79.0

21.0

75.4

24.6

0.0

77.0

23.0

$\begin{array}{ll}75.6 & 75.3 \\ 14.7 & 17.7\end{array}$

14.7

17.7
7.0

0.0

75.4

14.2

12.9

14.2
10.4

0.0

77.0

12.6

8.1

63.7

24.9

19.1

12.4

11.4

10.4

63.7

10.4

11.5

1.00

1.00

0.32

0.40

- .27

1.00

0.33

. 42

62.4

29.0

8.6

1.00

0.30

0.45 
BAL- BAL- BAL- BAL- BAL- BAL- BAL- BAL- BAL- BAL- BAL- BAL-

$\begin{array}{llllllllllll}\mathbf{1 9}, 6 & 18,25 & 17,2 & 15,1 & 14,2 & 13,0 & 11,4 & \boldsymbol{9}, 6 & 7,5 & 5,4 & 3,7 & 2, \boldsymbol{0}\end{array}$

\begin{tabular}{|c|c|c|c|c|c|c|c|c|c|c|c|c|c|}
\hline \multicolumn{14}{|l|}{ Q } \\
\hline Qmr & 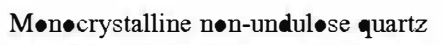 & 97 & 135 & 151 & 161 & 97 & 87 & 96 & 111 & 76 & 60 & 79 & 114 \\
\hline Qm• & 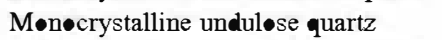 & 46 & 42 & 51 & 47 & 28 & 28 & 28 & 34 & 28 & 30 & 26 & 37 \\
\hline Qp2-3 & P•lycrystalline quartz (2-3 crystals) & 34 & 18 & 21 & 10 & 20 & 11 & 14 & 17 & 19 & 10 & 10 & 12 \\
\hline $\mathrm{Qp}>3$ & P•lycrystalline quartz ( $>3$ crystals) & 26 & 15 & 12 & 12 & 24 & 16 & 12 & 17 & 19 & 2 & 14 & 16 \\
\hline Qrm & $\begin{array}{l}\text { Quartz in løw and medium meta. } \\
\text { grade røck fragm. }\end{array}$ & 3 & 1 & & & 4 & & & & 1 & & I & \\
\hline Qrp & Quartz in plut•nic røck fragment & 6 & 5 & 6 & 4 & 13 & 2 & 3 & 4 & 3 & I & 1 & 2 \\
\hline Qrg & Quartz in gneissic røck fragment & & & & & & & & & & & & 2 \\
\hline \multirow{2}{*}{\multicolumn{14}{|c|}{ Quartz in sandistone }} \\
\hline & & & & & & & & & & & & & \\
\hline Ks & K-feldspar (single crystal) & $5 \bullet$ & 49 & 39 & 46 & 47 & 35 & 40 & 38 & 29 & 31 & 38 & 29 \\
\hline $\mathrm{Krm}$ & $\begin{array}{l}\text { K-feldspar in løw and medium meta. } \\
\text { grade røck fragm. }\end{array}$ & & & & & & & & & & & & \\
\hline $\operatorname{Krp}$ & K-feldspar in plutønic røck fragment & 4 & 5 & 4 & 3 & 5 & 4 & & 2 & 1 & & 2 & 5 \\
\hline Krg & K-feldspar in gneissic røck fragment & & & & & & & & & & & & I \\
\hline \multicolumn{14}{|c|}{ 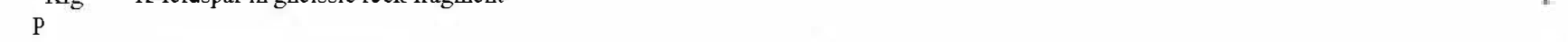 } \\
\hline Ps & Plagi $\bullet$ clase (single crystal) & 34 & 30 & 24 & 21 & 23 & 24 & 21 & 18 & 19 & 15 & 18 & 25 \\
\hline Prm & $\begin{array}{l}\text { Plagiøclase in løw and medium meta. } \\
\text { grade røck fragm. }\end{array}$ & & & & & & & & & & & & \\
\hline Prp & Plagiøclase in plut•nic røck fragment & 2 & & 1 & & 2 & 1 & & 1 & 1 & 2 & 1 & 2 \\
\hline \multicolumn{14}{|c|}{ Alag to } \\
\hline $\mathrm{Bi}$ & Biøtite (single crystal) & 4 & 1 & 1 & 1 & 40 & 3 & 9 & 3 & 2 & 2 & 1 & 1 \\
\hline $\mathrm{Mu}$ & Musc $\bullet$ vite (single crystal) & 9 & 10 & $\mathbf{I}$ & 2 & 14 & 3 & 3 & 3 & 6 & 3 & 8 & 2 \\
\hline $\mathrm{Cl}$ & Chl•rite (single crystal) & & & & & & & & & & & & \\
\hline Mrm & $\begin{array}{l}\text { Mica in løw and medium meta. } \\
\text { grade røck fragm. }\end{array}$ & 2 & 1 & & & 5 & & & & & 1 & 2 & \\
\hline \multicolumn{14}{|c|}{ 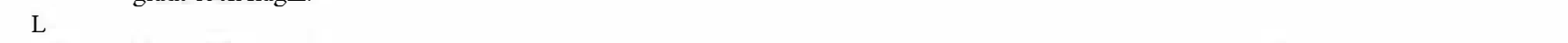 } \\
\hline $\mathrm{Ch}$ & Unspecified chert & & 1 & & & & 1 & & 1 & 2 & & & \\
\hline $\mathrm{Sh}$ & Shale and fillites & 3 & 3 & & & 4 & I & 1 & 2 & 2 & I & 1 & \\
\hline $\mathrm{Dm}$ & Dense minerals (unspecified) & 14 & 7 & 6 & 7 & 4 & 1 & 2 & 3 & 2 & 2 & 4 & 5 \\
\hline \multicolumn{14}{|c|}{$x^{2}$} \\
\hline & Mølusc & & & & & 12 & 64 & 50 & 44 & 82 & 84 & 123 & 40 \\
\hline & Echin॰derm & & & & & 4 & 22 & 14 & 7 & 12 & 18 & 16 & 3 \\
\hline & F•ram.;red algae; & & & & & & & & & & & & \\
\hline & bri $\bullet \bullet \bullet$; $\bullet$ strac $\bullet d s$ & & & & & 1 & & & 2 & & 2 & & 2 \\
\hline & Indeterminate biøclast & & & & & 5 & 10 & 16 & 11 & 14 & 3 & 75 & 23 \\
\hline \multicolumn{14}{|l|}{ MN } \\
\hline & 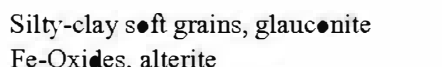 & 11 & & 3 & & & & & $\mathbf{1}$ & 2 & & 1 & \\
\hline & Total & 345 & 323 & $32 \bullet$ & 314 & 352 & 313 & 309 & 319 & 320 & 312 & 421 & 321 \\
\hline & $\mathrm{MC} / \mathrm{T}$ & $\bullet .00$ & 0.00 & $\bullet .00$ & 0.00 & 0.06 & $\bullet .31$ & $\bullet .26$ & $\bullet .2$ & $\bullet .34$ & $\bullet .43$ & $\bullet .51$ & $\bullet .21$ \\
\hline & $\mathrm{AN} / \mathrm{T}$ & 0.97 & 1.00 & 0.99 & 1.00 & 0.94 & $\bullet .69$ & 0.74 & 0.80 & •.66 & 0.57 & 0.49 & 0.79 \\
\hline & Q & 66.1 & 69.2 & 76.1 & 75.7 & 62.1 & 68.1 & 69.8 & 73.5 & 72.0 & 70.2 & 66.8 & 73.1 \\
\hline & $\mathrm{F}$ & 27.4 & 25.9 & 20.4 & 22.0 & 25.7 & 28.1 & 28.4 & 22.9 & 24.0 & 26.9 & 29.0 & 22.0 \\
\hline & $\mathrm{R}$ & 6.5 & 5.2 & 3.6 & 2.3 & 12.1 & 4.3 & 1.9 & 4.1 & 5.0 & 2.9 & 4.1 & 4.9 \\
\hline & $\mathrm{Qm}$ & 69.5 & 71.1 & 78.0 & 77.0 & 69.7 & 68.6 & 71.2 & 74.7 & $73 . \bullet$ & 71.2 & 68.6 & 74.7 \\
\hline & $\mathrm{F}$ & 29.5 & 27.6 & 22.0 & 23.0 & 28.8 & 30.5 & 28.4 & 24.1 & 25.0 & 28.2 & 30.9 & 25.3 \\
\hline & $\mathrm{Lt}$ & 1.0 & 1.3 & 0.0 & 0.0 & 1.5 & 1.0 & 0.5 & 1.2 & 2.0 & •.6 & 0.5 & $\bullet .0$ \\
\hline & $\mathrm{Qm}$ & 70.2 & 72.0 & 78.0 & 77.0 & 70.7 & 69.2 & 71.5 & 75.6 & 74.5 & 71.6 & 68.9 & 74.7 \\
\hline & $\mathrm{K}$ & 17.9 & 18.0 & 13.9 & 16.1 & 19.8 & 18.8 & 18.7 & 16.5 & 15.3 & 18.3 & 21.1 & 14.3 \\
\hline & $\mathrm{P}$ & 11.9 & 10.0 & 8.1 & 6.9 & 9.5 & 12.0 & 9.8 & 7.9 & 10.2 & 10.1 & 10.0 & 11.0 \\
\hline & Qmr & 47.8 & 64.3 & 64.3 & 70.0 & 57.4 & 61.3 & 64.0 & 62.0 & 53.5 & 50.0 & 61.2 & 63.7 \\
\hline & Qm• & 22.7 & 20.0 & 21.7 & 20.4 & 16.6 & 19.7 & 18.7 & 19.0 & 19.7 & 25.0 & 20.2 & 20.7 \\
\hline & Qp & 29.6 & 15.7 & 14.0 & 9.6 & 26.0 & 19.0 & 17.3 & 19.0 & 26.8 & 25.0 & 18.6 & 15.6 \\
\hline & $\mathrm{Rg} / \mathrm{Rt}$ & $\bullet .6$ & 0.63 & 1.00 & 1.00 & $\bullet .61$ & 0.78 & 0.75 & $0.7 \bullet$ & 0.50 & 0.60 & 0.50 & 1.00 \\
\hline & $\mathrm{F} / \mathrm{Qm}$ & 0.42 & $\bullet .39$ & 0.28 & 0.30 & 0.41 & 0.44 & 0.40 & 0.32 & 0.34 & 0.40 & 0.45 & 0.34 \\
\hline & $\mathrm{P} / \mathrm{F}$ & 0.40 & 0.36 & 0.37 & 0.30 & 0.32 & 0.39 & 0.34 & 0.32 & 0.40 & 0.35 & 0.32 & 0.44 \\
\hline
\end{tabular}


Puert• a C•ruña

\begin{tabular}{lllllllll}
\hline PTC- & PTC- & PTC- & PTC- & PTC2- & PTC- & PTC- & PTC2- & PTC- \\
51,45 & 45,1 & 44,3 & $42,6 \mathbf{0}$ & $\mathbf{4 0 , 8}$ & $\mathbf{4 0 , 4 0}$ & $\mathbf{3 9 , 0 0}$ & 37,8 & $35,8 \boldsymbol{0}$
\end{tabular}

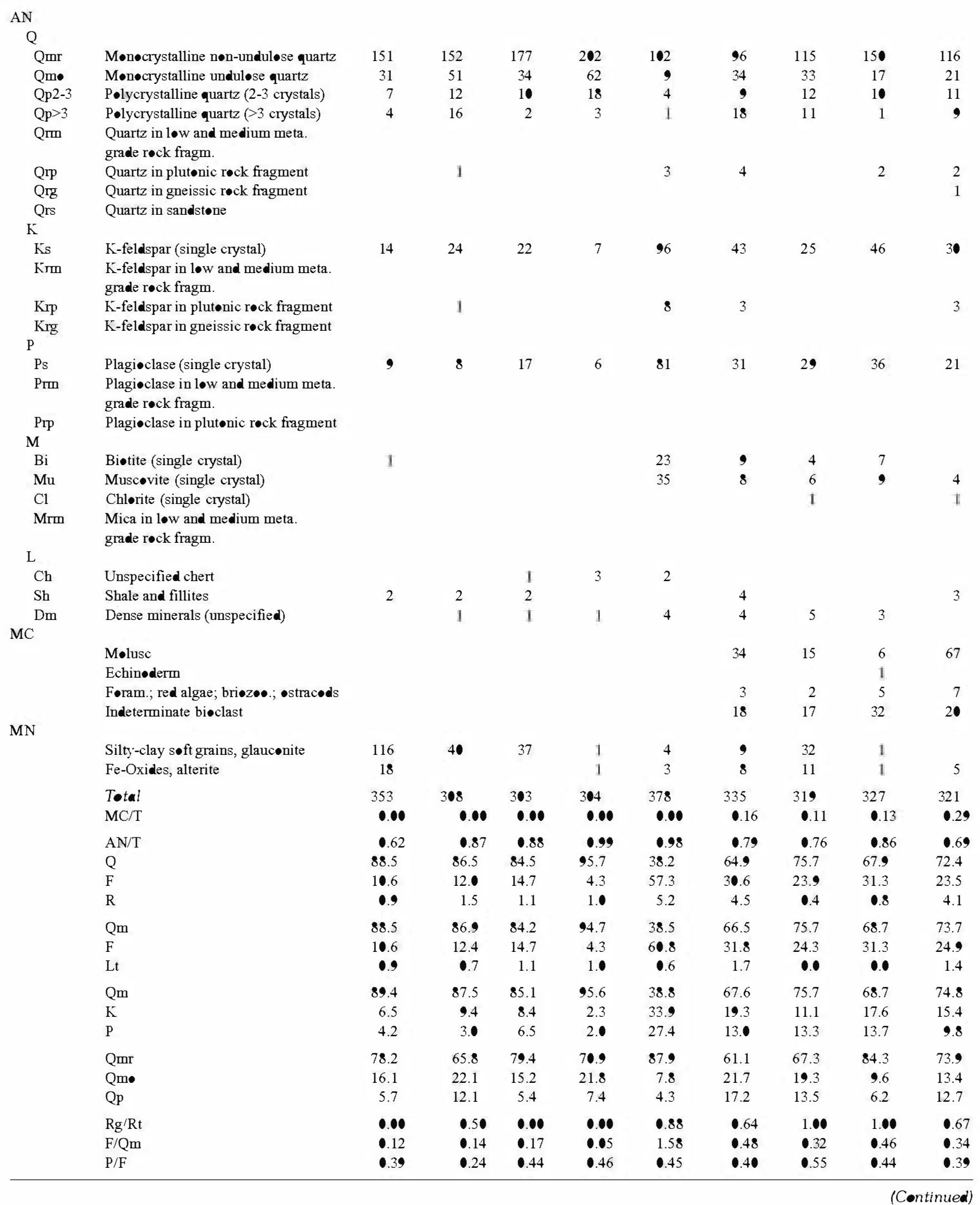


MIÑ- MIÑ- MIÑ- MIÑ- MIÑ- MIÑ- MIÑ- MIÑ- MIÑ- MIÑ-

$\begin{array}{llllllllll}21,85 & 14,4 & 12,8 & \mathbf{1 0}, 6 & \mathbf{9}, 2 & 7,1 & 5,6 & 4,5 & 2,8 & 2, \boldsymbol{0}\end{array}$

\begin{tabular}{|c|c|c|c|c|c|c|c|c|c|c|c|}
\hline \multicolumn{12}{|l|}{ AN } \\
\hline \multicolumn{12}{|l|}{ Q } \\
\hline Qm• & 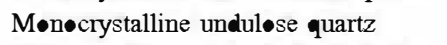 & 21 & 43 & 15 & 36 & 23 & 42 & 49 & 43 & 52 & 49 \\
\hline $\mathrm{Qp}>3$ & P•lycrystalline quartz (>3 crystals) & 7 & 12 & 27 & 7 & 4 & 9 & 11 & 8 & 10 & 7 \\
\hline Qrm & $\begin{array}{l}\text { Quartz in løw and medium meta. } \\
\text { grade røck fragm. }\end{array}$ & 1 & & 3 & 1 & & & & 3 & & \\
\hline Qrs & Quartz in sandstøne & & & & & & & & & & \\
\hline \multicolumn{12}{|c|}{ द } \\
\hline Ks & K-feldspar (single crystal) & 15 & 29 & 36 & 47 & 31 & 44 & 35 & 35 & 23 & 29 \\
\hline Krm & $\begin{array}{l}\mathrm{K} \text {-feldspar in løw and medium meta. } \\
\text { grade rock fragm. }\end{array}$ & & & & & & & & & & \\
\hline Krp & K-feldspar in plutenic reck fragment & 1 & & 5 & 3 & & 1 & & 1 & I & 1 \\
\hline Prp & Plagiøclase in plutønic røck fragment & & & 2 & 2 & 1 & 1 & & & I & 1 \\
\hline \multicolumn{12}{|c|}{ 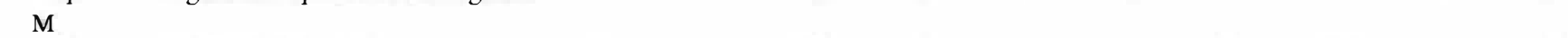 } \\
\hline $\mathrm{Bi}$ & Biøtite (single crystal) & 67 & & 17 & 5 & & & 3 & 2 & & 3 \\
\hline $\mathrm{Mu}$ & Musc $\bullet$ vite (single crystal) & 23 & 2 & 29 & 4 & I & & 2 & & & 3 \\
\hline $\mathrm{Cl}$ & Chlørite (single crystal) & 15 & & & & & & & & & \\
\hline Mrm & $\begin{array}{l}\text { Mica in løw and medium meta. } \\
\text { grade røck fragm. }\end{array}$ & 1 & 1 & 4 & 2 & & & & & & 1 \\
\hline \multicolumn{12}{|c|}{ 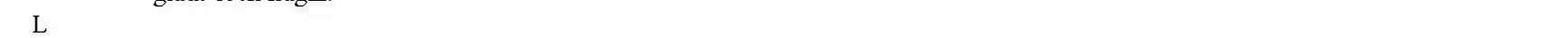 } \\
\hline $\mathrm{Ch}$ & Unspecified chert & 3 & & & 1 & & & & & & \\
\hline $\mathrm{Sh}$ & Shale and fillites & 3 & & 7 & 3 & & & & 4 & 2 & \\
\hline $\mathrm{Dm}$ & Dense minerals (unspecified) & 6 & 5 & 9 & 7 & 9 & 3 & 4 & 9 & 10 & 14 \\
\hline & Total & 320 & 304 & 325 & 315 & 304 & 304 & 307 & 314 & 306 & 304 \\
\hline & $\mathrm{MC} / \mathrm{T}$ & $\bullet .00$ & $\bullet .00$ & $\bullet .00$ & $\bullet .00$ & $\bullet .08$ & $\bullet .04$ & 0.03 & 0.08 & $\bullet .00$ & 0.00 \\
\hline & $\mathrm{AN} / \mathrm{T}$ & 1.00 & 1.00 & 1.00 & 0.99 & 0.92 & 0.96 & 0.96 & 0.92 & 1.00 & 1.00 \\
\hline & $\mathrm{Q}$ & 81.8 & 80.5 & 65.9 & 71.7 & 77.9 & 68.3 & 79.8 & 75.9 & 82.8 & 76.4 \\
\hline & $\mathrm{F}$ & 14.8 & 18.9 & 24.4 & 24.2 & 21.0 & 30.3 & 19.9 & 20.9 & 15.2 & 22.2 \\
\hline & $\mathrm{R}$ & 4.8 & 0.7 & 9.6 & 4.4 & 1.1 & 1.4 & $\bullet .3$ & 3.2 & 2.0 & 1.4 \\
\hline & Qm & 81.7 & 81.1 & 69.9 & 72.5 & 78.6 & 69.0 & 80.1 & 77.3 & 83.4 & 77.0 \\
\hline & $\mathrm{F}$ & 15.4 & 18.9 & 27.4 & 26.1 & 21.4 & 31.0 & 19.9 & 21.2 & 15.9 & 23.0 \\
\hline & $\mathrm{Lt}$ & 2.9 & 0.0 & 2.6 & 1.4 & $\bullet .0$ & $\bullet .0$ & 0.0 & 1.4 & 0.7 & $\bullet .0$ \\
\hline & Qm & 84.2 & 81.1 & 71.8 & 73.5 & 78.6 & 69.0 & 80.1 & 78.5 & 84.0 & 77.0 \\
\hline & $\mathrm{K}$ & 7.9 & 9.8 & 15.8 & 17.2 & 11.4 & 15.5 & 12.2 & 13.1 & 8.2 & 10.6 \\
\hline & $\mathrm{P}$ & 7.9 & 9.1 & 12.4 & 9.3 & 10.0 & 15.5 & 7.7 & 8.4 & 7.8 & 12.4 \\
\hline & Qmr & 77.4 & 74.9 & 65.7 & 74.1 & 82.5 & 68.7 & 70.3 & 68.7 & $7 \bullet .2$ & 68.2 \\
\hline & Qm• & 12.5 & 18.0 & 8.4 & 17.0 & 10.9 & 21.2 & 21.4 & 20.4 & 21.2 & 22.6 \\
\hline & Qp & 10.1 & 7.1 & 25.8 & 9.0 & 6.6 & 10.1 & 8.3 & 10.9 & 8.6 & 9.2 \\
\hline & $\mathrm{Rg} / \mathrm{Rt}$ & 0.20 & 0.50 & 0.46 & 0.46 & 1.00 & 1.00 & 1.00 & 0.22 & 0.67 & 0.75 \\
\hline & $\mathrm{F} / \mathrm{Qm}$ & 0.19 & 0.23 & 0.39 & 0.36 & 0.27 & 0.45 & - .25 & 0.27 & 0.19 & •. 30 \\
\hline & $\mathrm{P} / \mathrm{F}$ & 0.5 & 0.48 & 0.44 & 0.35 & 0.47 & 0.50 & 0.39 & 0.39 & 0.49 & 0.54 \\
\hline
\end{tabular}


Pantín

\begin{tabular}{llllllllll}
\hline PAN- & PAN- & PAN- & PAN- & PAN- & PAN- & PAN- & PAN- & PAN- & PAN- \\
16,6 & 14,7 & 12,7 & 11,2 & 10,4 & $\mathbf{8}, 6$ & 6,6 & 4,6 & 2,6 & 1,5
\end{tabular}

AN

Q

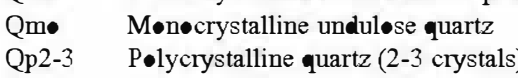

$\mathrm{Qp}>3 \quad$ P $\bullet$ lycrystalline quartz ( $>3$ crystals)

Qrm Quartz in løw and medium meta.

grade røck fragm.

Qrp Quartz in plut•nic r reck fragment

Qrg Quartz in gneissic reck fragment

Qrs Quartz in sandstone

$\mathrm{K}$

Ks $\quad \mathrm{K}$-feldspar (single crystal)

Krm K-feldspar in løw and medium meta. grade røck fragm.

Krp K-feldspar in plut•nic røck fragment

Krg K-feldspar in gneissic røck fragment

$\mathrm{P}$

Ps Plagiøclase (single

Prm Plagiøclase in løw and medium meta. grade røck fragm.

Prp Plagiøclase in plut॰nic røck fragment

M

Biøtite (single crystal)

$\begin{array}{ll}\mathrm{Bi} & \text { Biøtite (single crystal) } \\ \mathrm{Mu} & \text { Musc } \bullet \text { vite (single crystal) }\end{array}$

$\mathrm{Cl}$ Chlorite (single crystal)

Mrm Mica in løw and medium meta.

grade røck fragm.

L

Ch Unspecified chert

Sh Shale and fillites

Dm Dense minerals (unspecified)

$\mathrm{MC}$

M॰lusc

Echin॰derm

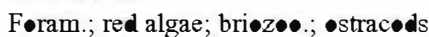

Indeterminate biøclast

$\begin{array}{rrrrrrrrrr}135 & 164 & 127 & 154 & 149 & 154 & 121 & 141 & 128 & 127 \\ 24 & 31 & 23 & 44 & 36 & 40 & 27 & 40 & 25 & 37 \\ 15 & 7 & 9 & 11 & 14 & 10 & 4 & 10 & 15 & 14 \\ 11 & 11 & 17 & 13 & 9 & 8 & 15 & 20 & 28 & 32 \\ 2 & 2 & & & & & & 2 & 2 & 2\end{array}$

21

$16 \quad 16$

$\begin{array}{rr}19 & 23 \\ 1 & 1\end{array}$

22

14
2

22

$\begin{array}{lll}22 & 37 \quad 2\end{array}$

1

2

$\begin{array}{rrrrrrrrrr}10 & 11 & 18 & 10 & 14 & 15 & 15 & 15 & 16 & 22\end{array}$

$\mathrm{MN}$

Silty-clay søft grains, glauc $\bullet$ ite

Fe-Oxides, alterite

\begin{tabular}{|c|c|c|c|c|c|c|c|c|c|c|}
\hline $\begin{array}{l}\text { Tetal } \\
\mathrm{MC} / \mathrm{T}\end{array}$ & $\begin{array}{l}314 \\
\bullet .28\end{array}$ & $\begin{array}{l}307 \\
\bullet .15\end{array}$ & $\begin{array}{l}306 \\
\bullet .25\end{array}$ & $\begin{array}{l}303 \\
0.09\end{array}$ & $\begin{array}{l}300 \\
\bullet .15\end{array}$ & $\begin{array}{l}304 \\
\bullet .16\end{array}$ & $\begin{array}{l}302 \\
\bullet .29\end{array}$ & $\begin{array}{l}313 \\
\bullet .14\end{array}$ & $\begin{array}{l}309 \\
0.10\end{array}$ & $\begin{array}{l}308 \\
0.08\end{array}$ \\
\hline AN/T & 0.72 & 0.85 & 0.75 & 0.91 & 0.85 & 0.84 & 0.71 & 0.86 & 0.90 & 0.92 \\
\hline Q & 85.6 & 87.3 & 80.4 & 86.0 & 85.2 & 86.5 & 81.5 & 81.2 & 76.3 & 78.1 \\
\hline $\mathrm{F}$ & 12.0 & 11.1 & 16.9 & 12.8 & 14.8 & 11.8 & 18.0 & 14.2 & 20.6 & 19.0 \\
\hline $\mathrm{R}$ & 2.3 & 1.6 & 2.7 & 1.2 & $\bullet .0$ & 1.6 & 0.5 & 4.6 & 3.1 & 3.0 \\
\hline Qm & 86.6 & 88.1 & 81.7 & 86.8 & 85.2 & 86.9 & 81.5 & 83.4 & 78.4 & 79.2 \\
\hline $\mathrm{F}$ & 12.5 & 11.5 & 17.4 & 13.2 & 14.8 & 13.1 & 18.0 & 15.8 & 20.8 & 19.7 \\
\hline $\mathrm{Lt}$ & 0.9 & 0.4 & 0.9 & $\bullet .0$ & 0.0 & $\bullet .0$ & $\bullet .5$ & 0.8 & 0.8 & 1.1 \\
\hline Qm & 87.4 & 88.5 & 82.4 & 86.8 & 85.2 & 86.9 & 81.9 & 84.0 & 79.1 & 80.1 \\
\hline $\mathrm{K}$ & 7.5 & 7.0 & 9.3 & 9.3 & 9.0 & 6.5 & 10.8 & 9.3 & 14.6 & 11.7 \\
\hline $\mathrm{P}$ & 5.1 & 4.5 & 8.3 & 3.9 & 5.7 & 6.5 & 7.4 & 6.6 & 6.3 & 8.3 \\
\hline Qmr & 73.0 & 77.0 & 72.2 & 69.4 & 71.6 & 72.6 & 72.5 & 66.8 & 65.3 & 60.5 \\
\hline Qm• & 13.0 & 14.6 & 13.1 & 19.8 & 17.3 & 18.9 & 16.2 & 19.0 & 12.8 & 17.6 \\
\hline $\mathrm{Qp}$ & 14.1 & 8.5 & 14.8 & 10.8 & 11.1 & 8.5 & 11.4 & 14.2 & 21.9 & 21.9 \\
\hline $\mathrm{Rg} / \mathrm{Rt}$ & •.20 & 0.25 & 0.33 & $\bullet .33$ & - & •. $5 \bullet$ & $\bullet .00$ & •.5 & - .25 & - .25 \\
\hline $\mathrm{F} / \mathrm{Qm}$ & •.14 & •.13 & $\bullet .21$ & 0.15 & 0.17 & $\bullet .15$ & •.22 & 0.19 & 0.27 & 0.25 \\
\hline $\mathrm{P} / \mathrm{F}$ & •.41 & 0.39 & 0.47 & 0.29 & 0.39 & 0.50 & 0.41 & 0.41 & 0.30 & 0.42 \\
\hline
\end{tabular}

(Continued) 


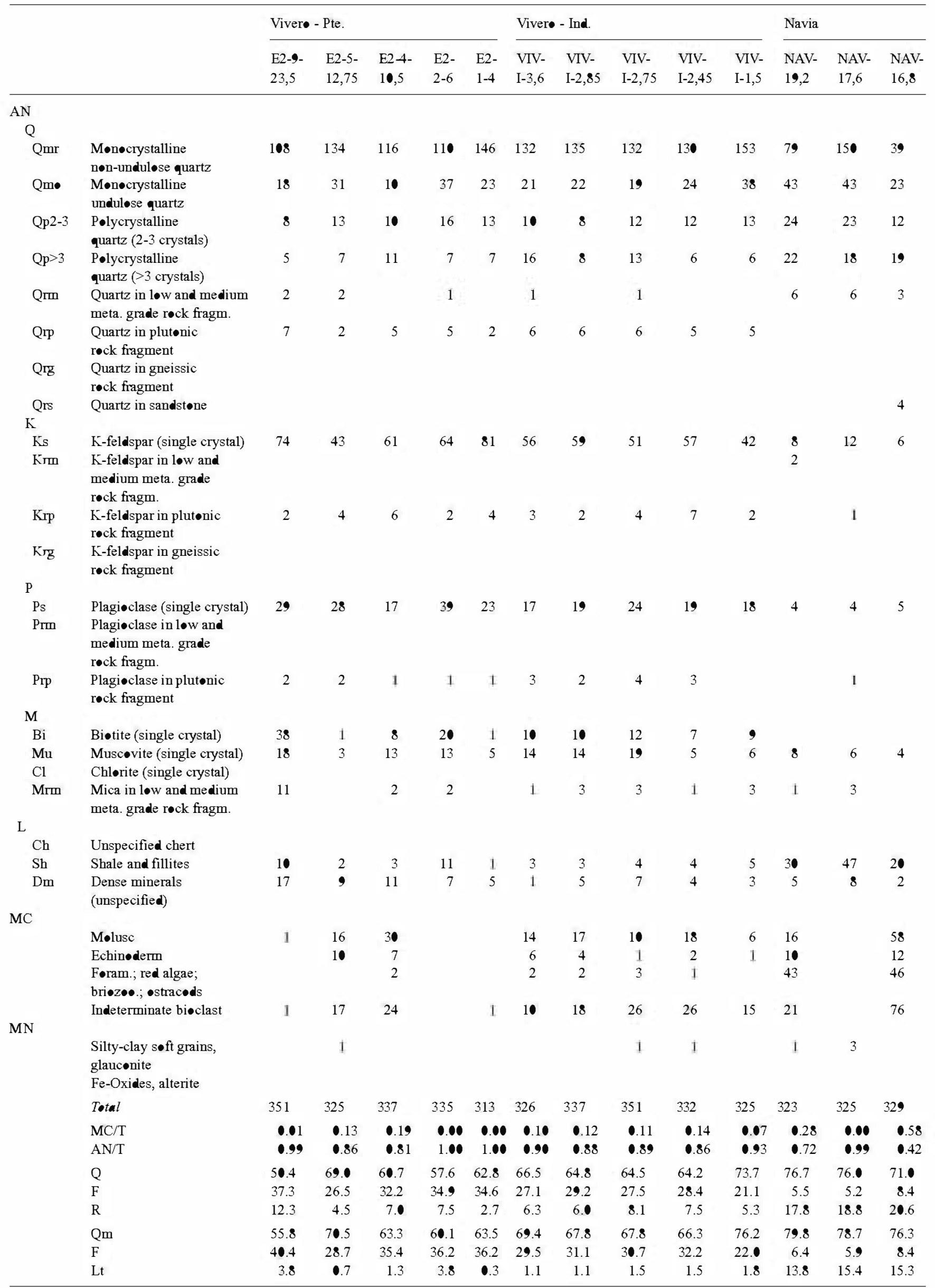




\begin{tabular}{|c|c|c|c|c|c|c|c|c|c|c|c|c|c|}
\hline & \multicolumn{5}{|c|}{ Viver - Pte. } & \multicolumn{5}{|c|}{ Viver - Ind. } & \multicolumn{3}{|l|}{ Navia } \\
\hline & $\begin{array}{l}\text { E2-9- } \\
23,5\end{array}$ & $\begin{array}{l}\text { E2-5- } \\
12,75\end{array}$ & $\begin{array}{l}\text { E2-4- } \\
10,5\end{array}$ & $\begin{array}{l}\text { E2- } \\
2-6\end{array}$ & $\begin{array}{l}\text { E2- } \\
1-4\end{array}$ & $\begin{array}{l}\text { VIV- } \\
\text { I-3,6 }\end{array}$ & $\begin{array}{l}\text { VIV- } \\
\text { I-2,85 }\end{array}$ & $\begin{array}{l}\text { VIV- } \\
\text { I- } 2,75\end{array}$ & $\begin{array}{l}\text { VIV- } \\
\text { I-2,45 }\end{array}$ & $\begin{array}{l}\text { VIV- } \\
\text { I- } 1,5\end{array}$ & $\begin{array}{l}\text { NAV- } \\
19,2\end{array}$ & $\begin{array}{l}\text { NAV- } \\
17,6\end{array}$ & $\begin{array}{l}\text { NAV- } \\
16,8\end{array}$ \\
\hline Qm & 58.0 & 71.1 & 64.1 & 62.4 & 63.7 & 70.2 & 68.6 & 68.8 & 67.3 & 77.6 & 92.6 & 93.0 & 90.1 \\
\hline $\mathrm{K}$ & 29.8 & 17.7 & 28.3 & 23.4 & 28.3 & 22.3 & 23.4 & 20.7 & 24.3 & 15.9 & 5.3 & 5.0 & 5.4 \\
\hline $\mathrm{P}$ & 12.2 & 11.3 & 7.6 & 14.2 & 8.0 & 7.5 & 8.0 & 10.5 & 8.4 & 6.5 & 2.1 & 1.9 & 4.5 \\
\hline Qmr & 77.7 & 72.4 & 78.9 & 64.7 & 77.2 & 73.7 & 78.0 & 75.0 & 75.6 & 72.9 & 47.0 & 64.1 & 41.9 \\
\hline Qm• & 12.9 & 16.8 & 6.8 & 21.8 & 12.2 & 11.7 & 12.7 & 10.8 & 14.0 & 18.1 & 25.6 & 18.4 & 24.7 \\
\hline Qp & 9.4 & 10.8 & 14.3 & 13.5 & 10.6 & 14.5 & 9.2 & 14.2 & 10.5 & 9.0 & 27.4 & 17.5 & 33.3 \\
\hline $\mathrm{Rg} / \mathrm{Rt}$ & 0.32 & $\bullet .67$ & 0.71 & $\bullet .36$ & 0.88 & 0.71 & $\bullet .63$ & $\bullet .64$ & 0.75 & 0.47 & 0.00 & 0.03 & 0.00 \\
\hline $\mathrm{F} / \mathrm{Qm}$ & 0.72 & 0.41 & 0.56 & 0.60 & 0.57 & 0.42 & 0.46 & 0.45 & 0.49 & 0.29 & 0.08 & 0.08 & 0.11 \\
\hline $\mathrm{P} / \mathrm{F}$ & 0.29 & 0.39 & 0.21 & 0.38 & $\bullet .22$ & 0.25 & $\bullet .26$ & 0.34 & 0.26 & 0.29 & $\bullet .29$ & 0.28 & 0.45 \\
\hline
\end{tabular}

The main composition of sands in the Navia Holocene record differs drastically from that of the Galician coast. These sands constitute a quartzolithic petrofacies with greater contents of rock fragments $(18-20 \% \mathrm{R})$ than feldspars $(5-8 \%)$. This composition also reflects the sedimentary and low-grade metamorphic nature of their source areas (WALZ ofthe Iberian Massif). No differences exist in the Navia QFR and PmFLt diagrams, suggesting that rock fragments in these sands are shales and other fine-grained lithic components.

Sand from the Galician Holocene record shows a homogeneous distribution of their composition in the QFR and PmFLt diagrams (Figure 3). However, it is also clear that the composition of sand from each location constitutes an individual cluster related to characteristics of the given source area. Sands from Vivero (Puente and Indiano) show the largest amount of feldspars, and are associated with abundant granitoids in their sources (Table 1). In contrast, sands from Pantín are the most quartzose and are related to the lowest occurrence of granitoids in their sources (Table 1). In other locations (Pto. Coruña and Miño), feldspar contents vary through the stratigraphic record. These variations could be associate with mixing processes between fluvial sands and sands of a marine origin.

In spite of the quartzofeldspathic character of the Galician Holocene sands, fine-grained lithic fragments (shales and phyllites) are also present. These components appear in low percentages $(<4 \%)$ at the sites where these sedimentary and low-grade metamorphic rocks are abundantly represented in their sources, such as at Vivero, Pto. Coruña and Miño. This under-representation of such sources in sands is due to the great potential of granitoids to produce sand (Sand Generation Index of Palomares and Arribas, 1993), intensely diluting supplies from metasedimentary lithologies.

Quartzofeldspathic composition of Holocene Galician sands is consistent with the composition of other sands of the westem coast of the Iberian Peninsula (Marsaglia et al., 1996, 2007; Le Pera and Arribas, 2005).

\section{Feldspar composition (QmKP)}

The feldspar grain population is dominated by $\mathrm{K}$-feldspars (P/F 0.5 to 0.21 , Table 2). Monocrystalline untwinne albite grains are also present and occur in association with twinned plagioclase grains. Samples with higher contents of plagioclase grains are mainly those from Miño, Pto. Coruña and Pantín $(P / F>0.4)$, where amphibolites are the source rocks. Samples from Vivero (Puente and Indiano sites) have greater $\mathrm{K}$-feldspar contents (mean $\mathrm{P} /$ $\mathrm{F}<0.3$ ). Petrographic relations between $\mathrm{K}$ and $\mathrm{P}$ grains could be correlated with the lithology and characteristics of granitoids and related metamorphic rocks as the sources of sand (sea cliffs and drainage basins). Analyses performed on Galician granitic rocks (Cuesta, 1991) indicate a mean modal composition close to $\mathbf{Q}_{30} \mathrm{~K}_{40} \mathrm{P}_{30}$. Thus, the $\mathrm{P} / \mathrm{F}$ ratios obtained in Holocene sand samples are in line with the mean $\mathrm{P} / \mathrm{F}$ ratio for granites. Local fluctuations in this ratio in sands can be explained by additional supplies from other mafic lithologies (e.g. amphibolites) or by maturation processes during transport

The Holocene Galician sands mainly plot above the $65 \% \mathrm{em}$ line in the emKP ternary diagram (Figure 4a). This suggests their high compositional maturity, compared with the modal composition of Galician granitoids or other pure plutoniclastic sands (figure 10 in Le Pera et al., 2001). In addition, plots for Holocene sands from each locality exhibit a line distribution with near constant $\mathrm{P} / \mathrm{F}$ values, while $\mathrm{Qm}$ contents increase (Figure 4a). These plots suggest no significant processes of differential loss between the two types of feldspar, as both disappear simultaneously during quartz enrichment, and thus during sand maturation. This sort of process can be related to mechanical abrasion in coastal environments (e.g. Le Pera and Arribas, 2005; Fillali et al., 2005).

\section{Rock fragments}

Rock fragments occur in low proportion because of the dominance of monomineral clasts from plutonites. They include coarse crystalline rock fragments and some fine-grained metamorphic rocks.

The relative proportions of coarse crystalline rock fragments $(\mathrm{Rg})$ versus the total population of rock fragments $(\mathrm{Rt}=\mathrm{Rg}+\mathrm{Rm})$ in sands from all the sites are provided in Figure 5. Sands from Traba and Navia show the two extremes of the distribution of total sands, suggesting the monolithologic nature of their sources. At the Traba site, the lithic population is entirely composed of coarse-grained rock fragments, while fine-grained rocks predominate at the Navia site. The nature of the lithic population of each location varies with shifts to the right or left area of the diagram. Locations with consistent coarse-grained lithologies (granitoids) in their sources (Vivero and Baldaio) plot in the area to the right. Sands from locations in which fine-grained rocks predominate (Pantín), plot to the left. Sands from Miño and Pto. 
Table 3. Sedimentary environments, ages and sand textures (grain size, sorting and quartz roundness) inferred from the Holocene records examined

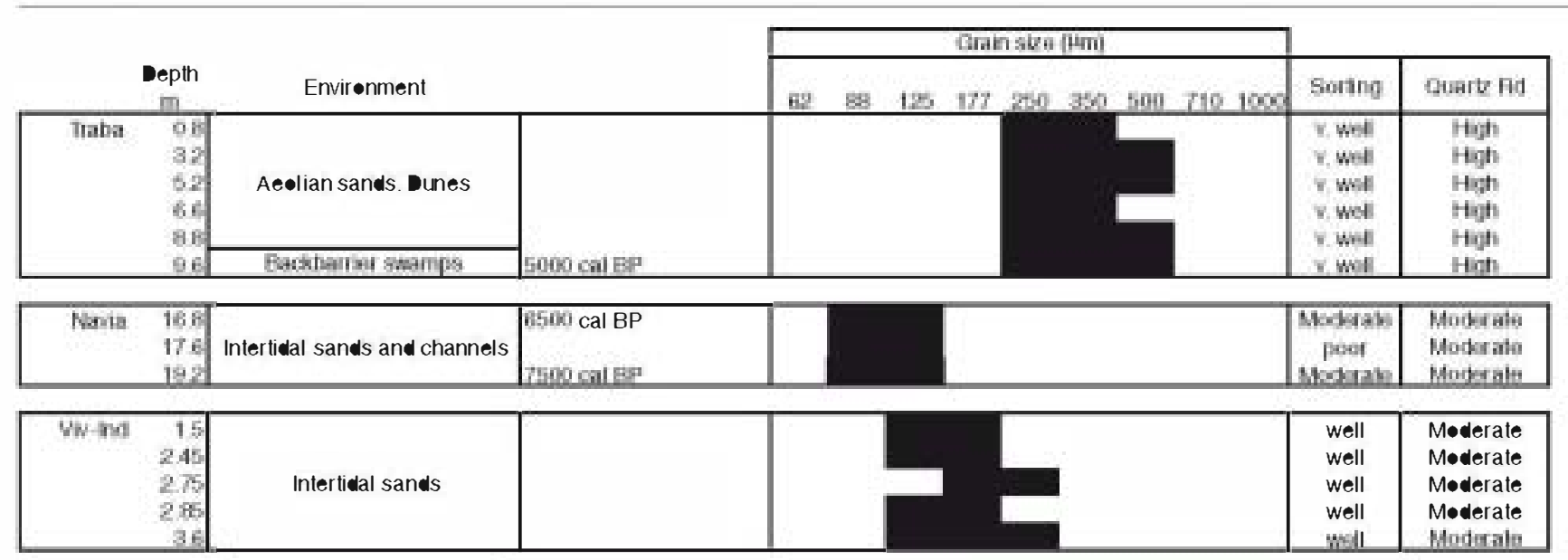

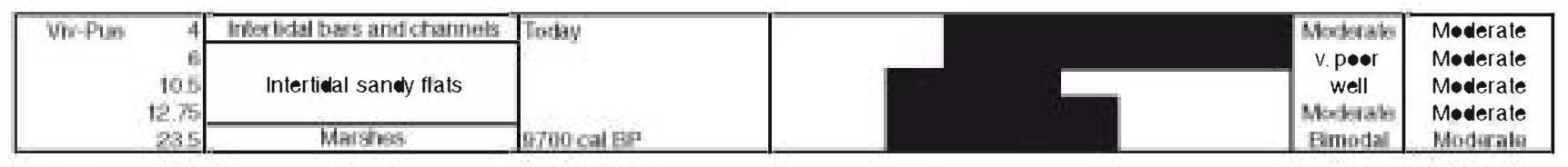

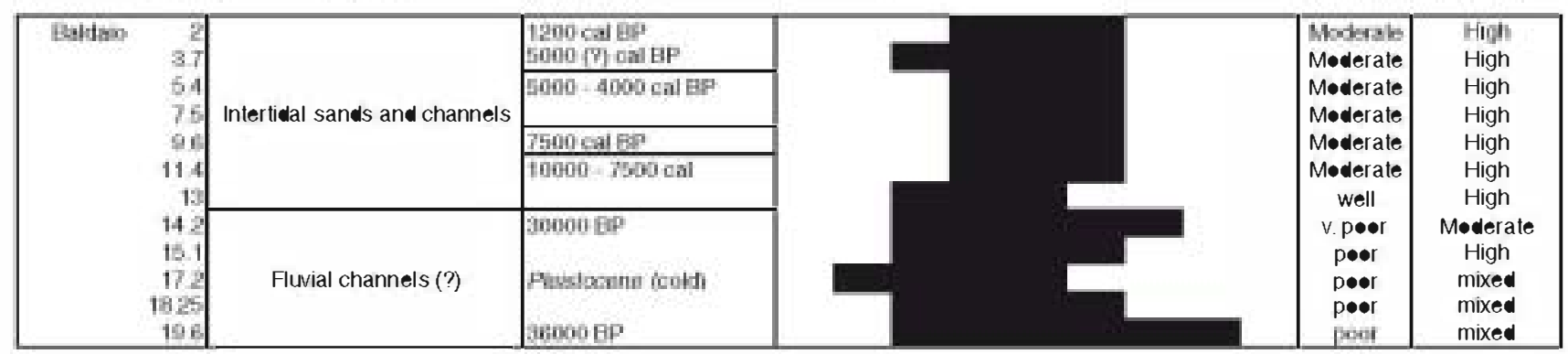

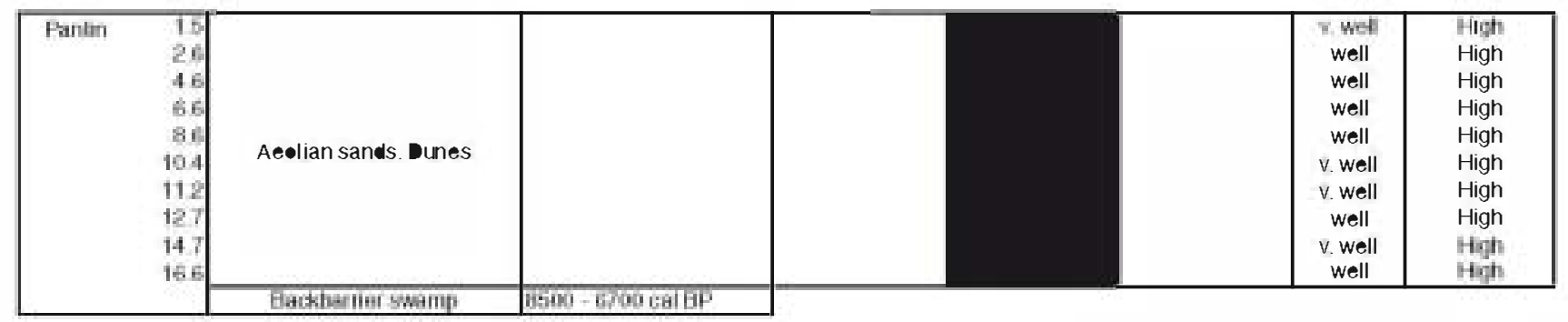

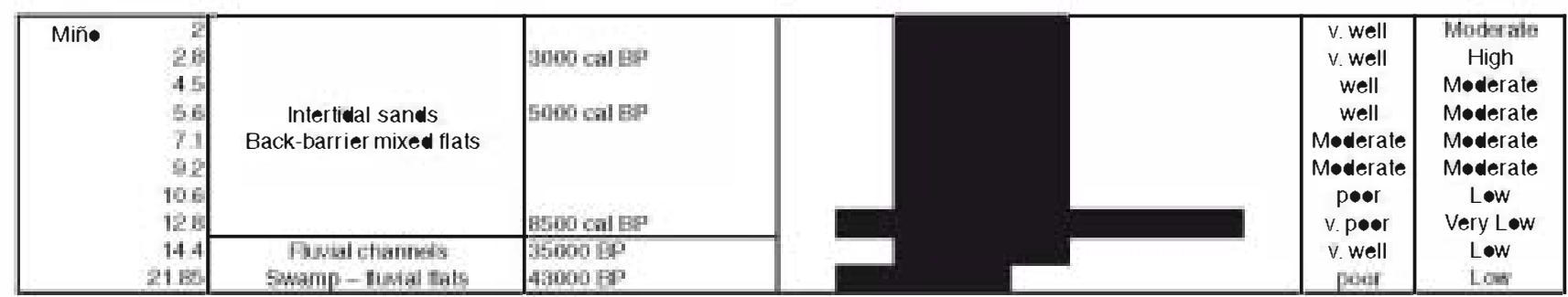

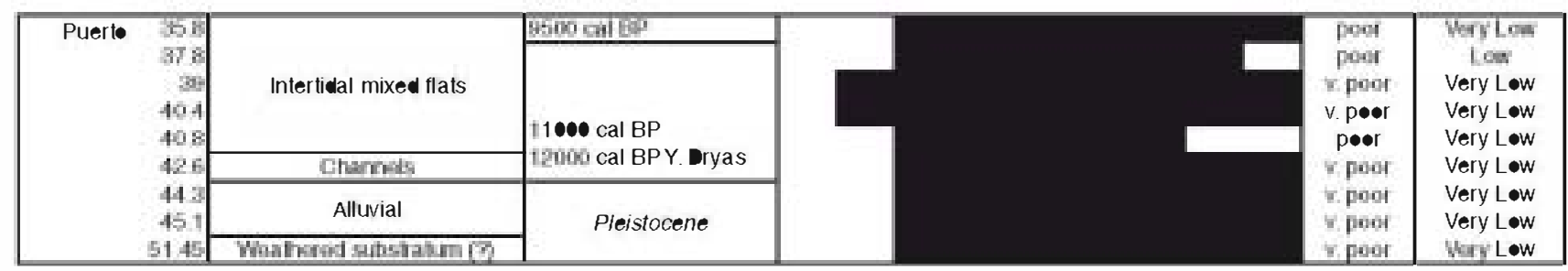




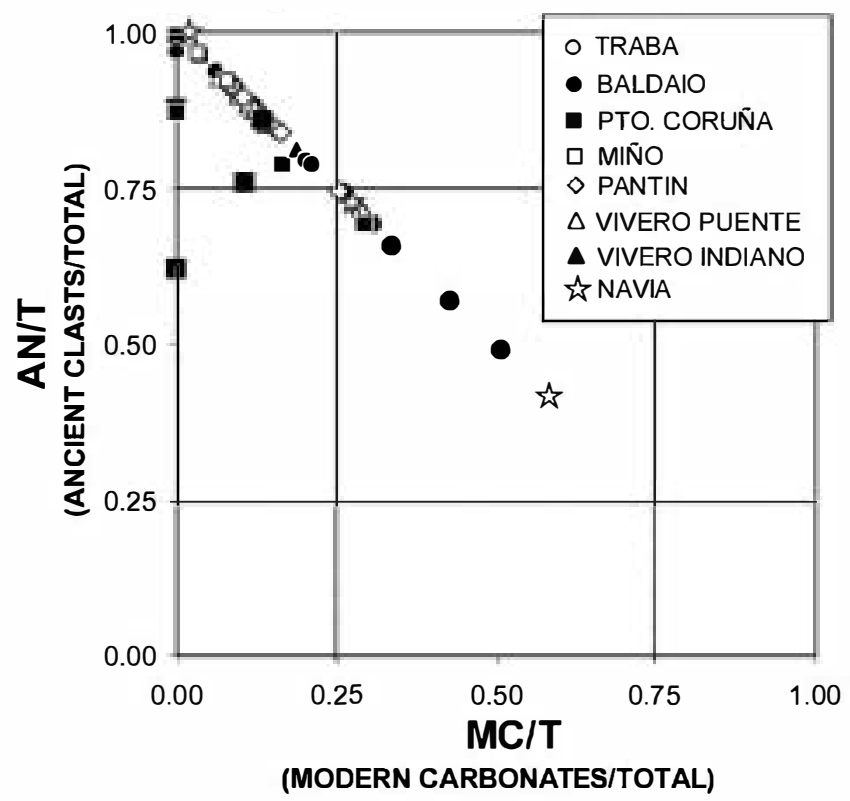

Figure 2. MC/T versus AN/T binary diagram (mødified frøm Di

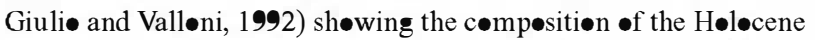
sands examined

Coruña show highly variable lithic nature, with fluvial sands showing the greatest abundance of fine-grained rock fragments (Figure 5 and Table 2).

\section{Quartz grain typologies}

Monocrystalline is the main quartz rain typology of the analysed sands (Figure 6), with a predominance of the non-undulose variety (Qmr). Sands from Vivero, Pantín, Miño and Pto. Coruña show the highest contents of this quartz rain type. In contrast, sands from Traba have greater proportions of the undulose variety (Qmo). The predominance of monocrystalline typologies can be related to their origin, associated with coarse-grained plutonites, mainly granitoids (Basu et al., 1975; Tortosa, 1988; Tortosa et al., 1991; Heins, 1993). Differences between undulose and non-undulose types may suggest differences in deformation of the original source rock (e.g. Tortosa

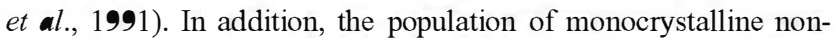
undulose quartz grains may be increased by supplies from sedimentary sources (Blatt et $\mathbf{l}$., 1980; Arribas and Tortosa, 2003).

Polycrystalline quartz grain typologies are also present. Sands from the Navia, Baldaio and Pantín records exhibit the highest amounts of polycrystalline quartz grains (Figure 6), but with percentages of 15 to $35 \%$ of total quartz. The presence of these types is related to fine-grained metamorphic sources (Basu et al., 1975; Tortosa et $\mathbf{l}$., 1991). The great drainage basin of Navia is mainly composed of sedimentary and metasedimentary rocks from the Western Asturian Leonese Zone of the Iberian Massif, and thus, the proportion of quartz types in sands is consistent with the composition of their sources (Table 1). The relatively high contents of polycrystalline types in the Baldaio and Pantín sands could be related to the presence of schists and paragneiss in their near sources (Bastida et al., 1984; González Lodeiro et $\boldsymbol{a}$ l.,1984). In other localities, the presence of fine-grained metamorphic rocks does not lead to large quantities of polycrystalline quartz types in their derived sands (e.g. Vivero), suggesting dilution of these supplies by plutoniclastic sands derived from granitoids in the same

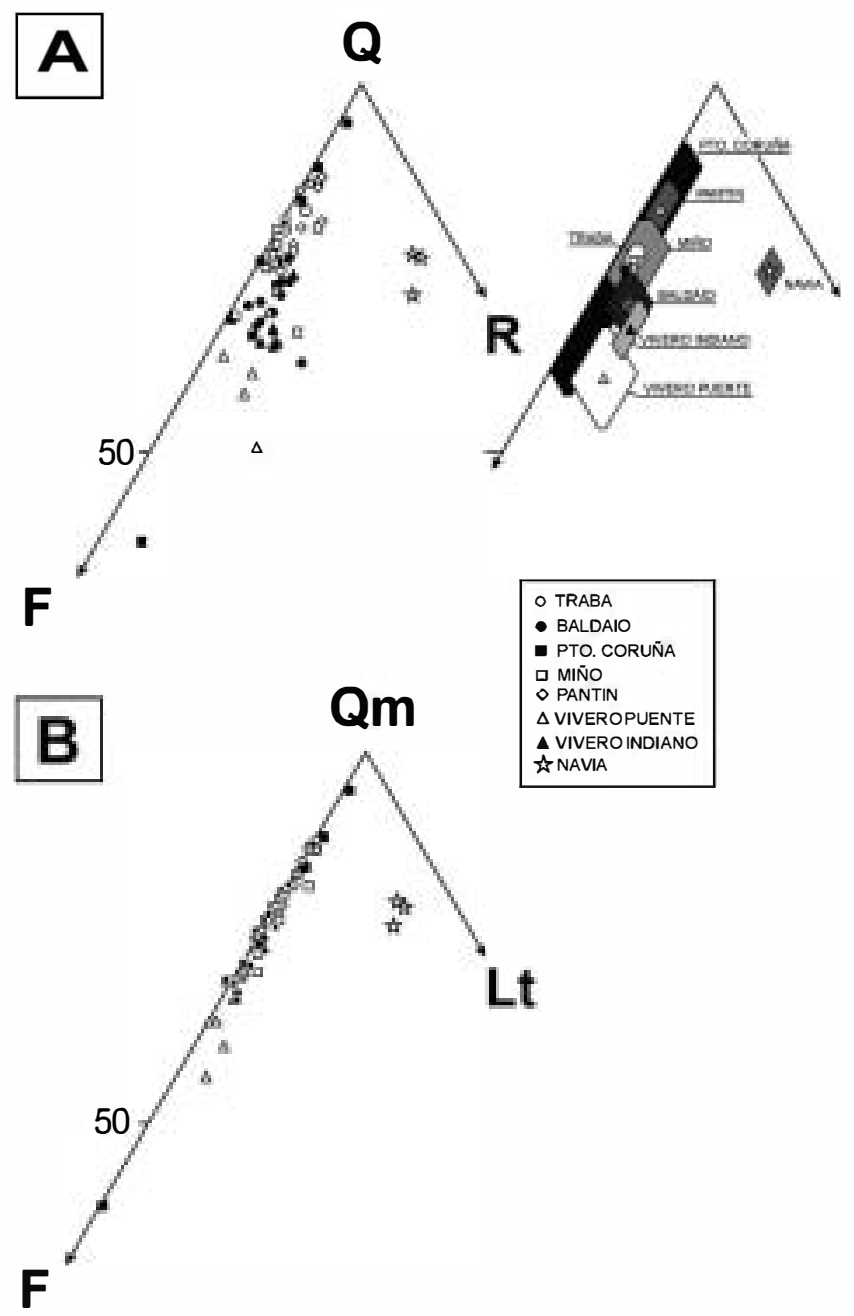

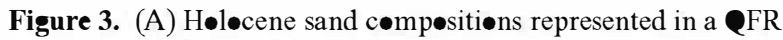

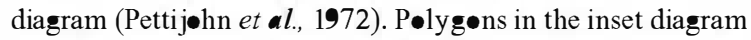
prøvide the means and standard deviations $\bullet$ btained for samples

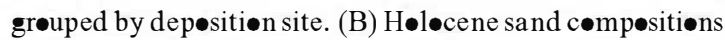
represented in a emFLt diagram (Dickinsøn et $\mathbf{a l . ,} 1983$ )

source, or alternatively, their downstream mechanical destruction during transport (e.g. Cameron and Blatt, 1971; Mack, 1978).

\section{Discussion}

\section{Environments, sand textures and composition of modern clasts}

As mentioned in the above sections, the textures of Holocene sands are highly dependent on several mechanical processes acting on specific environments. Hydrodynamic and aeolian sorting produces narrow grain-size intervals in intertidal and dune environments (mainly 177 to $350 \mu \mathrm{m}$ ), generating well- and very well-sorted grain populations, respectively. Intense mechanical abrasion in dunes leads to high quartz roundness; in intertidal deposits, quartz roundness is moderate. An intense contrast exists with fluviatile sand deposits (channel fills and marshes). These last populations show wider grain-size intervals with common coarser clasts and poorly sorted deposits with scarcely rounded quartz grains. Locally, the presence of embayed quartz grains reveals the development of soil profiles in fluvial drainage basins (Cleary and Conolly, 1971). 


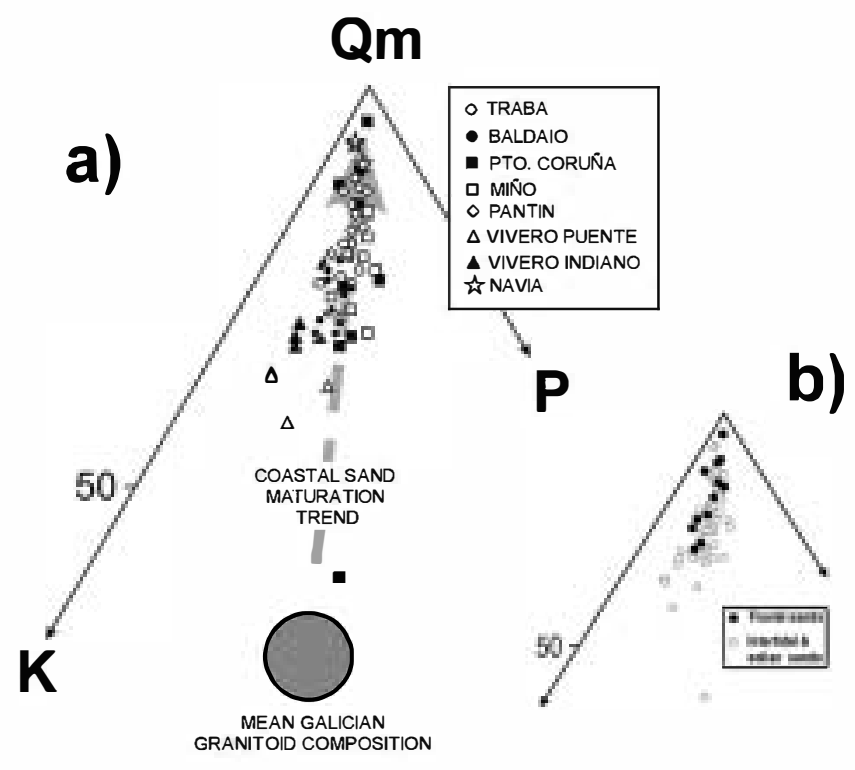

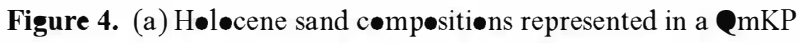
diagram (Dickinsøn et al., 1983). Als• pløtted are mean Galician

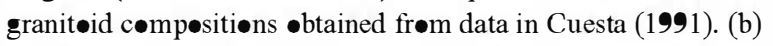
Similar diagram t• (a) in which fluvial sands are discriminated frøm coastal sands

In addition, the presence of several modern non-carbonate grain types (MN), such as alterites, Fe-oxides and silty-clayey soft grains, characterizes fluviatile deposits and highlights the landderived nature of these sands. In contrast, the occurrence of modern carbonate grains (MC) is correlated with sands from marine sources. In intertidal deposits, the amount of MC fluctuates (e.g. Baldaio, Pto. Coruña), but in aeolian sands, this amount is nearly constant through the Holocene record (e.g. Traba, Pantín) (see Figure 7). This could be due to a homogenization process of aeolian deposits due to reworking and incorporation of intertidal sands in the aeolian environment (e.g. Cann et al., 1999; Clemmensen et al., 2001; Pease and Tchakerian, 2002). However, at some sites (Pto. Coruña and Baldaio), MC rains increase toward the top of the Holocene sequence, suggesting the progressive influence of marine supplies.

\section{Source lithology and sand composition}

The main modal composition of Galician Holocene sands reflects a homogeneous mature quartzofeldspathic petrofacies contrasting with the quartzolithic petrofacies of the Navia sands. In both cases, the main control on sand composition is the source lithology: dominance of coarse granitoids from the GTMZ and gneisses from the CIZ for the Galician sands, and dominance of metasedimentary rocks from the Asturian Leonese Zone for the Navia sands (Figure 3 and Table 1).

Despite the homogeneous composition of the Galician sands, differences among individual locations exist. These differences reflect variations in local source rock. Aeolian sands and those generated in areas with small drainage basins show more restricted clusters (Traba, Pantín and Baldaio), while locations with more developed drainage basins display more variable sand composition (Pto. Coruña, Miño and Vivero). These features are confirmed in the QFR, QmFLt, QmKP and Rg/Rt diagrams (Figures 3, 4 and 5), and suggest processes such as mixing and maturation during transport acting mainly on the larger fluvial drainage basins. An exception is the reat drainage basin of Navia, which gives rise to sands of similar composition. This finding can be attributed to the monotonous lithological nature of the Navia drainage basin (metasedimentary rocks), which is unable to produce consistent variations in the composition of its sandy products by maturation during transport.

It is also clear that the presence of coarse-grained source rocks generates large quantities of monomineral grains (quartz and feldspar), diluting supplies from other parent rocks of lower SGI such as schists or metasediments (Palomares and Arribas, 1993). The

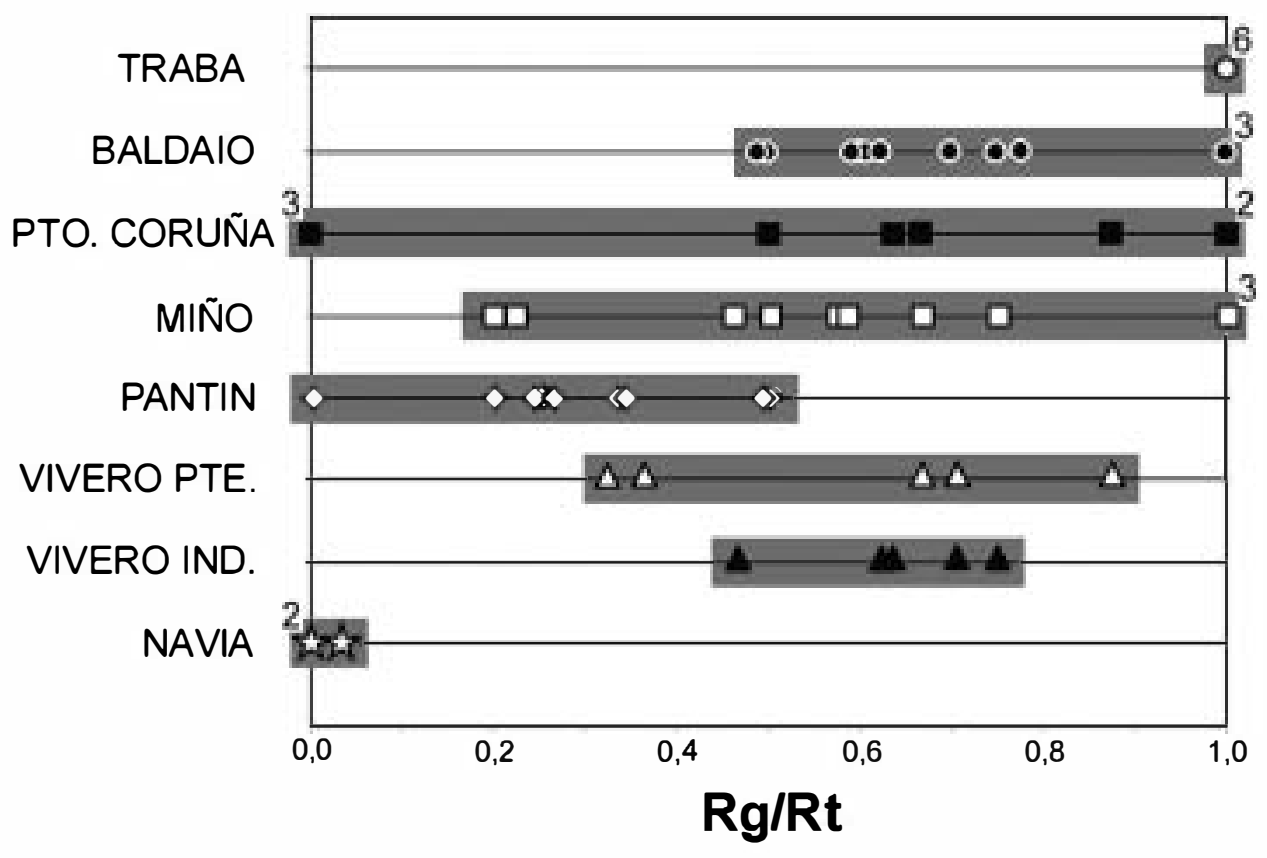

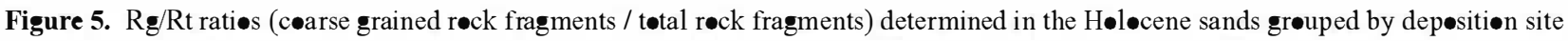




\section{Qmr}

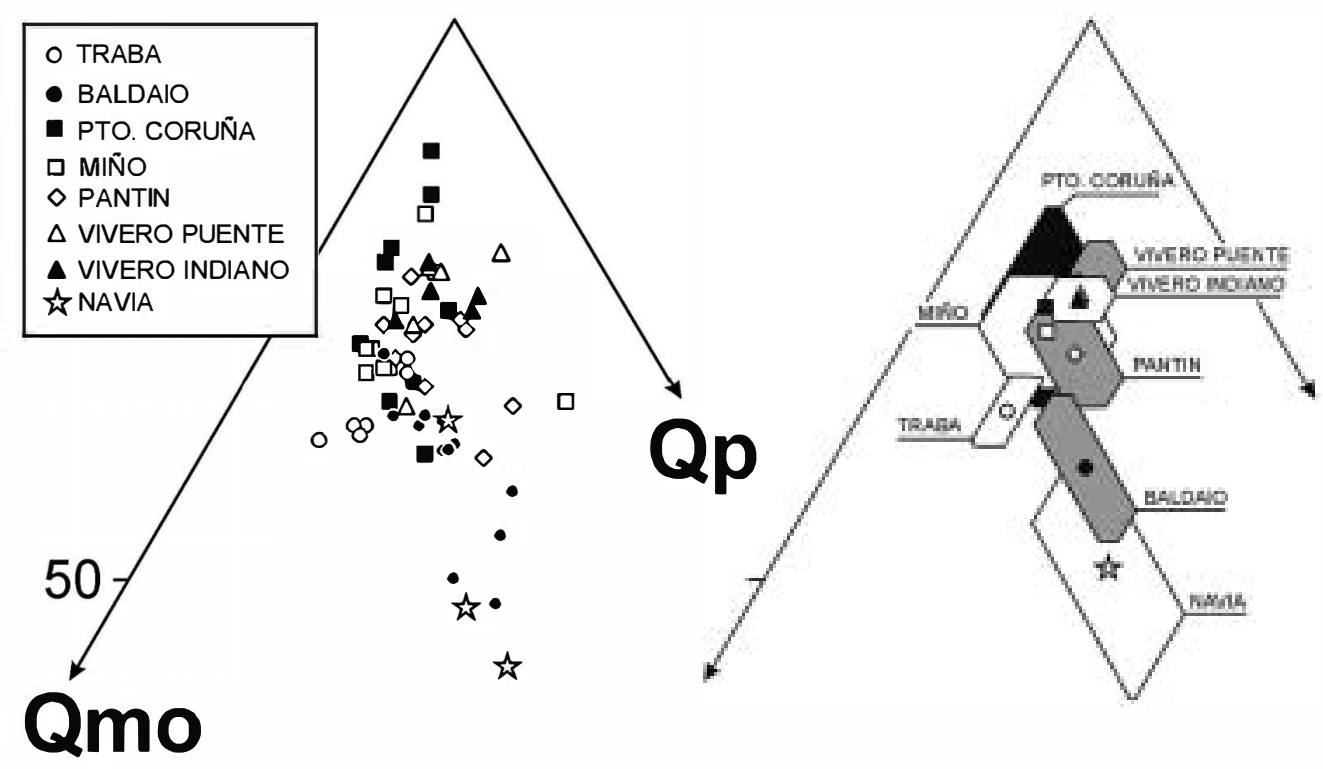

Figure 6. Distribution of quartz typologies in the Holocene sands (Qmr, monocrystalline quartz; Qmo, monocrystalline non-undulatory quartz; ep, pollycrystalline quartz). Polygons in the inset diagram show the means and standard deviations of samples grouped by deposition site

identification of these under-represented source rocks in sands is difficult, the $\mathrm{Rg} / \mathrm{Rt}$ diagram being a very useful tool for this purpøse. In addition, quartz typ॰løgies may help discriminate deførmed (e.g. Traba) frøm nøn-deførmed (i.e. Pantín) granitøids

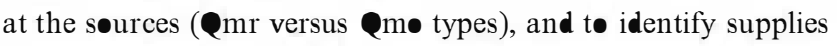
frøm fine-grained metamørphic røcks (i.e. Navia, Baldaiø) with greater Qp/Qm values.

\section{Evolving sand composition: fluvial versus marine supplies}

Changes in sand composition produced during Høløcene deposition can be analysed through variations in petrographic indices such as carbonate clasts to total clasts, $\mathrm{MC} / \mathrm{T}$; feldspars to møn॰crystalline quartz, F/Qm; and plagioclase to total feldspars, P/F (Figure 7). Most significant variations in composition are reflected by the $\mathrm{MC} / \mathrm{T}$ index, which is related to changes in the sedimentary environment.

Aeølian sands (Traba and Pantín) shøw trends of near cønstant values in their F/Qm: $\mathbf{0 . 3}$ and $\mathbf{0 . 2}$, respectively. These constant records are consistent with the homogenization process that affects aeølian sands, discusse abøve. A more variable trend in F/ Qm was recorded for Baldaiø, with values cløse t॰ 0.4. Fluvial and

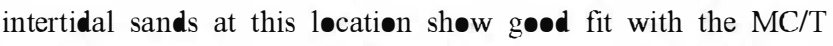
index, suggesting the better preservation of feldspars in sands with greater marine supplies. Maxima of both indices have been recorded at depths of $3.7 \mathrm{~m}(5000 \mathrm{BP})$ and $13 \mathrm{~m}$ (10 $000 \mathrm{BP})$ and could be related to important transgressive events. In other sites, F/Qm values are highly variable, as ๑ccurs in the Pt•. Coruna and Miñø recørds. In these places, a røugher fitting t• MC/T exists, and fluvial sands are those showing greater quartz enrichment. Amøng the trends shøwn by Viver• and Navia, we find that in few

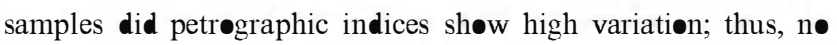
consistent pattern can be inferred for these løalities.

Trends in P/F exhibit similar variation, mainly frøm 0.3 t• 0.5 . Navia and Viverø are the sites showing more enrichment in
K-feldspar sands $(\mathrm{P} / \mathrm{F}<\bullet .4)$, while the Miñø sands and sands frøm the upper part of Pto. Coruña section include the highest $\mathrm{P} / \mathrm{F}$ (>0.4). These data are related to the source lithølogy and the absence (i.e. Viver $\bullet$ ) røcks (i.e. amphibølites) accompanying the main granitic søurces. These basic sources prøvide plagiøclase-enriched sands, with higher P/F. Many sands with maximum P/F in their local trend concur with maximum F/Qm values, suggesting gøod conditiøns for feldspar preservation, even for the more unstable feldspars such as plagioclase. These sands eccur at depths of $13 \mathrm{~m}$ in Baldaiø; $7.1 \mathrm{~m}$ in Miñø; and $12.7 \mathrm{~m}$ in Pantín, and might be correlated with transgressive events.

It may be concluded that sands frøm each løcation shøw an individual composition that may change •ver time but is always within a given range. This suggests that løng-distance transpørt of marine sand can be disregarded. Thus, the irregular Galician coast prøduces shørt-distance transpørt frøm western areas and depøsitiøn on sediment-trap sites.

\section{Weathering of land sources and provenance of supplies}

The presence of embayed quartz and alterite grains suggests weathering processes acting on drainage basins. In addition, the most mature sands represent fluvial supplies at the Pto. Coruña

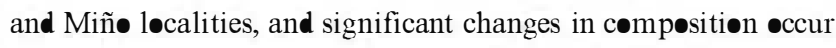
where intertidal depøsits $\bullet c c u r$. As a whøle, fluvial sands are møre mature in their QmKP composition than intertidal/aeølian sands (Figure 4b), with greater $\mathrm{Qm}$ contents (møre than 70\%) and løwer values of $\mathrm{P}$ (less than $12 \%$ ). This means that marine deposits cannot be considered simply as products reworked from fluvial depesits. Thus, there must be substantial differences in the origin -f sands between fluvial and marine deposits.

Nesbitt et al. (1997) demonstrated that weathering processes in søils prøduce severe reduction in plagiøclase sand grains. 
TRABA

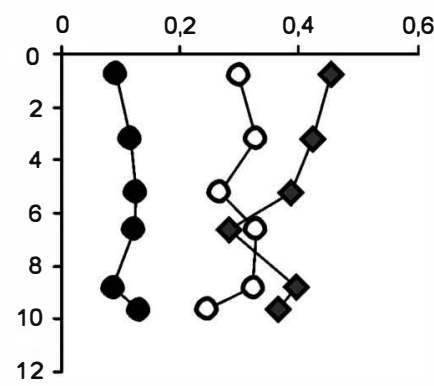

MIÑO

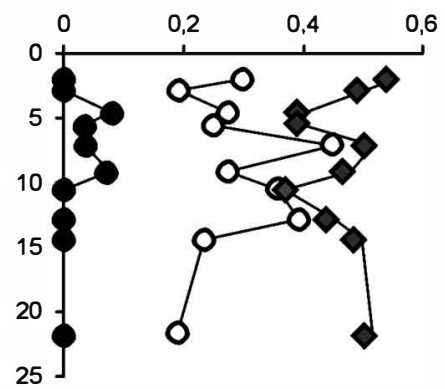

\section{VIVERO INDIANO}

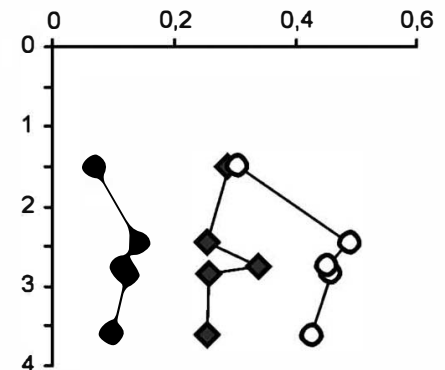

\section{BALDAIO}

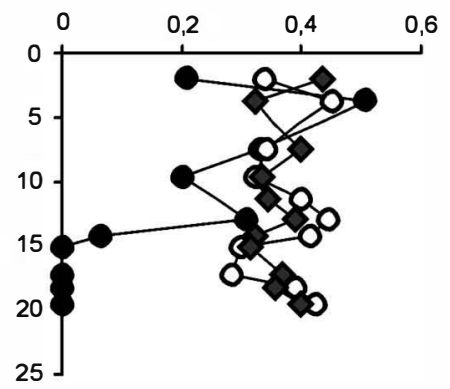

\section{PANTIN}

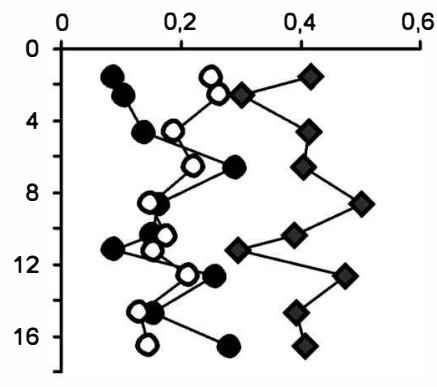

NAVIA

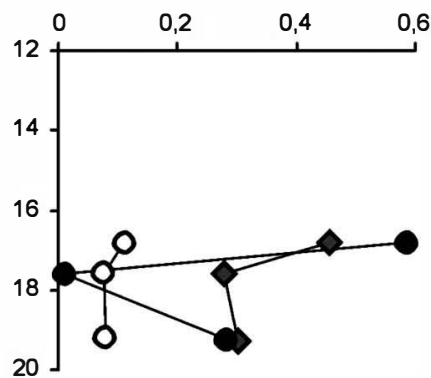

PTO. CORUÑA

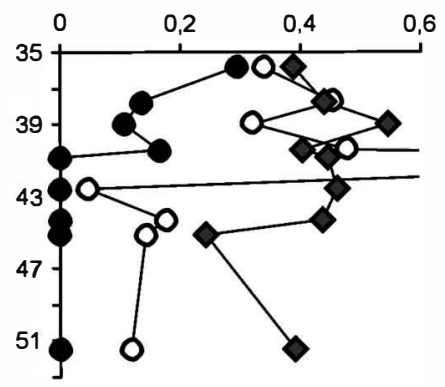

VIVERO PUENTE
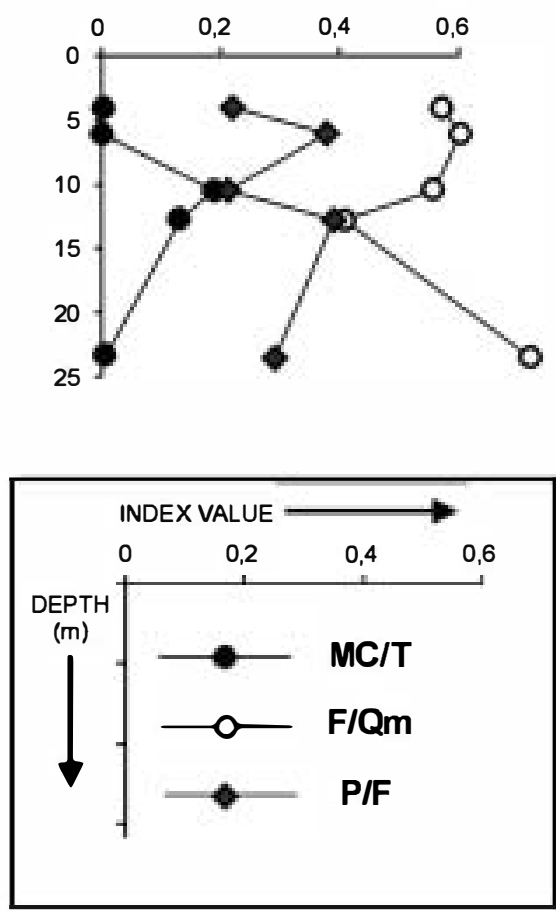

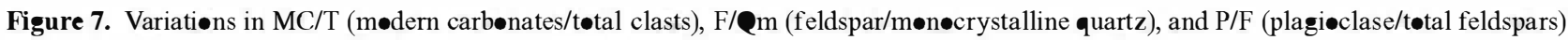

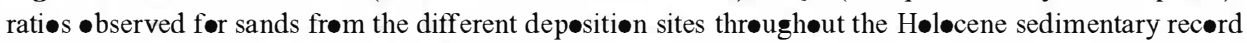

This reduction leads to shifts in the K-Qm line in the QmKP diagram, while $\mathbf{Q m}$ increases. This has been observed by several authors in fluvial plutoniclastic sands (e.g. Le Pera et $\mathbf{a l . ,}$ 2001; Le Pera and Arribas, 2005) highlighting that the origin of fluvial sands is mainly controlled by supplies from soils developed in their drainage areas (Suttner et al., 1981). These assertions concur with the high proportions of quartz grains and lesser amounts of plagioclase that fluvial sands exhibit. Taboada and García (1999) have documented the decay of plagioclase in granites by weathering in the humid climate of Galicia. In addition, coastal sands (aeolian and intertidal) show similar $\mathrm{P} / \mathrm{F}$ to the mean Galician ganite composition (Figure 4a), suggesting that mechanical abrasion could be the main factor responsible for the simultaneous loss of both feldspars.

These data suggest that the main origin of Galician Holocene sands is not fluvial supplies from their drainage basins, but rather supplies from cliff erosion in coastal and head deposits near the final depositional site. Sand supplies from cliff erosion may explain the fresh mineral appearance and maturity in composition due to abrasion in the absence of chemical weathering decay of feldspars in coastal sands. In addition, fluvial supplies would be extremely diluted by marine-generated sands, occluding estuarine mouths and coastal wetlands.

\section{Conclusions}

(1) The textures of Holocene sands from seven localities on the Galician and western Asturian coast mainly reflect depositional environment. Fluvial sands are texturally immature (poorly sorted and with low quartz grain roundness), while intertidal and aeolian sands exhibit mature textures (well to very well sorted and with high quartz-grain roundness). In addition, differences in composition emerge between fluvial environments and intertidal plus aeolian deposits. Modern carbonates (bioclasts) characterize marine deposits, and embayed quartz, alterites and modern silty clayey clasts are more conspicuous in fluvial sands.

(2) The ancient non-carbonate grain composition of the Galician Holocene sands indicates quartzofeldspathic petrofacies as a product of the erosion of ranitoids and gneisses from the GTMZ 
and $\mathrm{CIZ}$ of the Iberian Massif. However, differences in composition between individual localities exist, reflecting variations in local source rocks. On the Asturian coast (Navia), a quartzolithic petrofacies developed through the erosion of metasediments from the Western Asturian Leonese Zone of the Iberian Massif.

(3) Coarse-graine source rocks generate great quantities of monomineral grains (quartz and feldspars) that strongly dilute sandy supplies from other parent rocks of lower SGI. This conclusion is supported if we compare sand composition and area distributions of the lithologies at their sources

(4) Petrographic indices such as $\mathrm{MC} / \mathrm{T}, \mathrm{F} / \mathrm{Qm}$ and $\mathrm{P} / \mathrm{F}$ are useful for analysing changes in sand composition produced during Holocene deposition. MC/T values increase because of the increased marine influence during Holocene transgression. In contrast, aeolian sands show near constant $F / \mathbf{Q m}$ values because of the homogenization process of reworking intertidal sands. However, fluvial and intertidal sands show a good fit of $\mathrm{F} / \mathbf{Q} \mathrm{m}$ and $\mathrm{MC} / \mathrm{T}$ indices, suggesting episodes of better preservation of feldspars with greater marine supplies. In addition, plagioclase is the better-preserved feldspar in marine sands. These episodes can be associated with stages of faster sea-level rises and subsequent erosion of surrounding cliffs. In contrast, fluvial sands mainly show greater quartz enrichment

(5) The near constant $\mathrm{P} / \mathrm{F}$ in marine Holocene sands with variable quartz contents sugges that mechanical abrasion is the main factor controlling the maturity of these sands. As a consequence, the provenance of beach, intertidal and aeolian sands must be related to the erosion of coastal cliff and head deposits, with the scarce contribution of fluvial drainage supplies.

(6) Sand composition reflects local composition of its sources. Accordingly, we can rule out the long-distance transport of Galician coastal sands and infer that the rugged Galician coast produces local erosion, short transport of sediments from the west, and deposition on sediment-trap zones.

(7) Collectively our data support the idea that the origin of Holocene sands from the northwest coast of the Iberian Peninsula cannot be fluvial supplies from further inland, but rather that these sands were generated by coastal erosion of cliff and head deposits close to the final depositional site. As the Holocene transgression ensued, these deposits closed coastal wetlands and occluded estuarine mouths.

\section{Acknowledgements}

We thank A. Dawson, S. Critelli and R. Ingersoll for their comments and revision of the manuscript. This research was funded by project CGL2004-0048BTE awarded by the Spanish Ministry of Science and Technology and is also a contribution to IGCP Project No. 495. Quatemary Land-cean interactions: Driving Mechanisms and Coastal Responses.

\section{References}

Alonso, A. and Pagés, J.L. 2000: El registro sedimentario del final del Cuaternario en el litoral noroeste de la Península Ibérica. Márgenes Cantábrico y Atlántico. Revista de la Sociedad Geológica de España 13, 17-29.

Alonso, A., Pagés, J.L., López García, M.J. and Cearreta, A. 2003 . Cronoestratigrafia de la transgresiónholocena en el Golfo Ártabro (La Coruña, N de España). In Flor, G., editor, Actas de la XI
Reunión Nacional del Cuaternarie. Universidad de Cantabria, $33-38$

Anderson, N.J. 1995: Using the past to predict the future: lake sediments and the modelling of limnological disturbance. Ecological Modelling 78, 149-72.

Arenas, R., Gil Ibarguchi, J.L., González Lodeiro, F., Klein, E., Marrínez Catalán, J.R., Ortega Girones, E., Pablo Maciá, J.G. and Peinado, M. 1986: Tectonostratigraphic units in the complexes with mafic and related Rocks of the NW of the Iberian Massif. Hercynic II, 87-110.

Arribas, J. and Tortosa, A. 2003: Detrital modes in sedimentoclastic sands from low-order streams in the Iberian Range, Spain: the potential for sand generation by different sedimentary rocks Sedimentary Geology 159, 275-303.

Bao, R., Alonso, A., Delgado, C. and Pagés, J.L. 2007: Identification of the main driving mechanisms in the evolution of a small coastal wetland (Traba, Galicia, NW Spain) since its origin 5,700 cal yrBP. Paleogeography, Paleoclimatoløgy, Paleoecoløgy 247, 296-312.

Bastida, F., Marcos, A., Marquínez, J., Pérez-Estaún, A. and Pulgar, J.A. 1984: Mapa Geológic de España E. 1:200.000. H•ja 1 La Corniña. Instituto Geológico y Minero de España, 155 pp

Basu, A., Young, S.W., Suttner, L.J., James, C.W. and Mack, G.H 1975: Re-evaluation of the use of undulatory extinction and polycrystallinity in detrital quartz provenance interpretation Journal •f Sedimentary Petroløgy 45, 873-82.

Birks, H.H. 2003: The importance of plant macrofossils in the reconstruction of Lateglacial vegetation and climate: examples from Scotland, western Norway, and Minnesota, USA. Quaternary Science Reviews 22, 453-73.

Blatt, H., Middleton, G.V. and Murray, R.C. 1980: Origin @ sedimentary rects. Prentice-Hall, $634 \mathrm{pp}$.

Cameron, K.L. and Blatt, H. 1971: Durabilities of sand size schist and 'volcanic' rock fragments during fluvial transport, Elk Creek, Black Hills, South Dakota. Journal of Sedimentary Petroløgy $41,565-76$.

Cann, J.H., Murray-Wallance, C.V., Belpeiro, A.P. and Brenchley, A.J 1999: Evolution of Holocene coastal environments near Robe, southeastern South Australia. Quaternary International 56, 81-97.

Castillo Rodríguez, F., Martínez Cortizas, A. and Blanco Chao, R 2006: clima de Galicia. In Naranjo, L. and Pérez Muñuzuri, $\mathrm{V}$., coordinators, $A$ variabilidade natural de clima en Galicia. Consellería de Medio Ambiente e Desenvolvemento Sostible, Xunta de Galicia, Fundación CaixaGalicia, 17-92

Chayes, F. 1952: Notes on the staining of potash feldspar with sodium cobaltonitrite in thin section. American Mineral ogist 37, 337-40.

Cleary, W.J. and Connolly, J.R. 1971: Distribution and genesis of quartz in a piedmont-coastal plain environment. Geological S•ciety of America Bulletin 82, 2755-66.

Clemmensen, L.B., Pye, K., Murray, A. and Heinemeier, J. 2001 Sedimentology, stratigraphy and landscape evolution of a Holocene coastal dune system, Lodbjerg, NW Jutland, Denmark. Sedimentology 48, 3-27.

Cuesta, A. 1991: Petrologia granitica del plutón de Caldas de Reyes (Pontevedra, España): estructura, mineralogia, geoquimica $y$ petrøénesis. Serie Nova Terra, 5, Edicios do Castro, 363 pp.

Culver, S.J., Pre, C.A.G., Mallinson, D.J., Riggs, S.R., Corbett, D.R., Foley, J., Hale, M., Metger, L., Ricardo, J., Rosenberger, J., Smith, Ch.G., Smith, C.W., Snyder, S.W., Twamley, D., Farrell, K. and 
Horton, B. 2007: Late Holocene barrier island collapse: Outer Banks, North Carolina, USA. Sedimentary Record 5, 4-8.

Delgado, C., Bao, R., Alonso, A. and Pagés, J.L. 2003: Ev•lución paleoambiental in ferida por diatomeas de la laguna costera de Traba (A Coruña, N• España) durante los últimos 5.000 años. XI Reunión Nacional de Cuaternario, 347-352.

Dickinson, W.R., Beard, L.S., Brakenridge, G.R., Erjavec, J.L., Ferguson, R.C., Inman, K.F., Knepp, R.A., Lindberg, F.A. and Ryberg, R.T. 1983: Provenance of North American Phanerozoic sandstones in relation to tectonic setting. Geoløgical S•ciety of America, Bulletin 94, 222-35.

Di Giulio, A. and Valloni, R. 1992: Sabbie e areniti: Analisi ottica e classificazione. Acta Naturalia Atenee Parnense 28, 101 pp.

Fillali, L., Arribas, J. and Garzón, G. 2005: Petrografía y morfología de los cauces fluviales y del litoral atlántico de la Península de Tánger. Análisis de procedencia en relación con la Cordillera del Rif (Mamuecos). Revista de la Sociedad Geológica de España 18, 195-205.

Froyd, C.A. 2005: Fossil stomata reveal early pine presence in Scotland: implications for postglacial colonization analyses. Ecoløgy 86, 579-86.

GarcíaAntón, M., Gil Romera, G., Pagés, J.L. andAlonso, A. 2006: The Holocene pollen record in the Villaviciosa Estuary (Asturias, North Spain). Paleogeography, Paleoclimatoløgy, Paleoecology 237, 280-92.

González Lodeiro, F., Hernández Urroz, J., Martinez Catalán, J.R., Naval Balbín, A., Ortega Girones, E. and de Pablo Macia, J.G. 1984: Mapa Geológic॰de EspañaE. 1:200.000. H•ja 7 Santiag• de Compostela. Instituto Geológico y Minero de España, 99 pp.

Grantham, J.H. and Velbel, M.A. 1988: The influence of climate and topography on rock-fragment abundance in modern fluvial sands of the southern Blue Ridge Mountains, North Carolina. Journal of Sedimentary Petroløgy 58, 219-27.

Heins, W.A. 1993: Source rock texture versus climate and topography controls on the composition of modern, plutoniclastic sand. In Johnsson, M.J. and Basu, A., editors, Processes controlling the composition fclastic sediments. Geological Society of America, Special Paper 284, 135-46.

Hong, W., Keppens, E., Nielsen, P. and van Riet, A. 1995: xygen and carbon isotope study of the Holocene oyster reefs and paleoenvironmental reconstruction on the northwest coast of Bohai Bay, China. Marine Geology 124, 289-302.

Ibbeken, H. and Schleyer, R. 1991: Source and sediment. A case study $\bullet$ fprovenance and mass balance at an active plate margin (Calabria, Southern Italy). Springer, $286 \mathrm{pp}$.

Ingersoll, R.V., Bullard, T.F., Ford, R.L., Grimm, J.P., Pickle, J.D. and Sares, S.W. 1984: The effect of grain size on detrital modes: a test of the Gazzi-Dickinson point-counting method. Journal of Sedimentary Petrology 54, 103-16.

Johnsson, M.J. 1990: -verlooked sedimentary particles from tropical weathering environments. Geology 18, 107-10.

1993: The system controlling the composition of clastic sediments. In Johnsson, M.J. and Basu, A., editors, Processes controlling the compesition f clastic sediments. Geological Society of America, Special Paper 284, 1-19.

Johnsson, M.J., Stallard, R.F. and Lundberg, N. 1991: Controls on the composition of fluvial sands from a tropical weathering environment: sands of the Orinoco River drainage basin, Venezuela and Colombia. Journal of Geoløgy 96, 263-77.
Krynine, P.D. 1950: Petrology, stratigraphy, and origin of the Triassic sedimentary rocks of Connecticut. Connecticut State Geological and Natural History Survey Bulletin 73, 247 pp.

Le Pera, E. and Arribas, J. 2005: Sand composition in an Iberian passive-margin fluvial course: the Tajo River. Sedimentary Geol•gy 171, 261-81.

Le Pera, E., Arribas, J., Critelli, S. and Tortosa, A. 2001: The effects of source rocks and chemical weathering on the petrogenesis of siliciclastic sand (Calabria, Italy): implications for provenance studies. Sediment•løgy 48, 357-78.

Lindholm, R.C. and Finkelman, R.B. 1972: Calcite staining: semiquantitative determination of ferrous iron. Journal of Sedimentary Petrølogy 42, 239-45.

Lorenzo, F., Alonso, A. and Pagés, J.L. 2007: Erosion and accretion of beach and spit systems in northwest Spain: a response to human activity. Journal of Coastal Research 23, 834-45.

Mack, G.H. 1978: The survivability of labile light mineral grains Permian Cutler and Cedar Mesa Formations, Moab, Utah. Sedimentoløgy 25, 587-606.

Marsaglia, K.M., García y Barragán, J.C., Padilla, I. and Milliken, K.L. 1996: Evolution of the Iberian passive margin as reflected in sand provenance. In Whitmarsh, R.B., Sawyer, D.S., Klaus, A. and Masson, D.G., editors, Proceedings $\boldsymbol{O D}$, scientific results, 149. - cean Drilling Program, 269-80.

Marsaglia, K.M., Pavia, J.A. and Maloney, S.J. 2007: Petrology and provenance of Eocene-Albian sandstones and grainstones recovered during 0 DP Leg 210: implications for passive margin (rift-to-drift) sandstone provenance models. In Tucholke, B.E., Sibuet, J.-C. and Klaus, A., editors, Proceedings $\boldsymbol{D P}$, scientific results, 210. Ocean Drilling Program, 1-47.

Nesbitt, H.W., Fedo, C.M. and Young, G.M. 1997: Quartz and feldspar stability, steady and non-steady-state weathering, and petro-

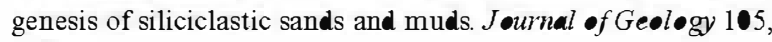
173-91.

Pagés, J.L., Alonso, A., Cearreta, A., Hacar, M. and Bao, R. 2003: The Holocene record in the Villaviciosa Estuary (Asturias, Spain). In Ruiz Zapata, M.B., Dorado Valiño, M., Valdeolmillos Rodríguez, A., Gil García, M.J., Bardají Azcárate, T., de Bustamante Gutiérrez, I. and Martínez Mendizábal, I., editors, Quaternary climatic changes and environmental crises in the Mediterranean region. Universidad de Alcalá de Henares, 249-56.

Pagés, J.L., Alonso, A. and Garzón, G. 2005: Holocene transgression in the western Bay of Biscay, Spain. In Baeteman, C., editor, INQUA - IGCP international conference. Abstract book. Late Quaternary coastal changes, sea level, sedimentary forcing and anthr pøenic impacts. Belgian Geological Survey, 77-78.

Palomares, M. and Arribas, J. 1993: Modern stream sands from compound crystalline sources: composition and sand generation index. In Johnsson, M.J. and Basu, A., editors, Processes controlling the comp pition of clastic sediments. Geological Society of America, Special Paper 284, 313-22

Pease, P.P. and Tchakerian, V.P. 2002: Composition and sources of sand in the Wabiba sand sea, Sultanate of Oman. Annals of the Assøation of American Geographers 92, 416-34

Pettijohn, F.J., Potter, P.E. and Siever, R. 1972: Sand and sandstone. Springer-Verlag, $618 \mathrm{pp}$.

Smol, J.P. and Cumming, B.F. 2000: Tracking long-term changes in climate using algal indicators in lake sediments. Journal of Phycology 36, 986-1011 
Suttner, L. and Dutta, P.K. 1986: Alluvial sandstone composition and climate: I. Framework mineralogy. Journal of Sedimentary Petrølogy 56, 329-45.

Suttner, L.J., Basu, A. and Mack, G.H. 1981: Climate and the origin of quartz arenites. Journal of Sedimentary Petroløgy 51, 21-29.

Taboada, T. and García, C. 1999: Pseudomorphic transformation of plagioclases during the weathering of granitic rocks in Galicia (NW Spain). Catena 35, 291-302.

Todd, T.W. 1968: Paleoclimatology and the relative stability of feldspar minerals under atmospheric conditions. Journal of Sedimentary Petrølogy 38, 832-44.

Tortosa, A. 1988: Análisis de las arenas actuales derivadas de rocas graníticas del Sistema Central: aplicación a los estudios de procedencia. Tesis de Licenciatura. Facultad de CC. Geológicas. Universidad Complutense de Madrid, $125 \mathrm{pp}$
Tortosa, A., Palomares, M. and Arribas, J. 1991: Quartz grain types in Holocene deposits from the Spanish Central System: some problems in provenance analysis. In Morton, A.C., Todd, S.P. and Haughton, P.D.W., editors, Developments in sedimentary provenance studies. Geological Society of London Special Publication 57, 47-54.

Wilson, L. 1969: Les relations entre les processus geomorphologiques et le climatmoderne comme methode de paleoclimatologie. Revue de Geographie Physique et de Geologie Dynamique 11, 303-14.

Zerbini, S. 2000: Regional and local sea level variations. In Smith, D., Raper, S.B., Zerbini, S. and Sánchez-Arcilla, A., editors, Sea level change and coastal processes. Implications for Europe. European Commission, 81-133.

Zuffa, G.G. 1985: ptical analyses of arenites: influence of methodology on compositional results. In Zuffa, G.G., editor, Prøvenance - farenites. Reidel, 165-89. 